\title{
Global existence of solutions for interval-valued second-order differential equations under generalized Hukuhara derivative
}

\author{
Van Hoa Ngo ${ }^{1 *}$ and Dinh Phu Nguyen ${ }^{2}$
}

"Correspondence:

ngovanhoa_clt@yahoo.com ' Division of Applied Mathematics, Ton Duc Thang University, Nguyen Huu Tho Street, District 7, Ho Chi Minh City, Vietnam

Full list of author information is available at the end of the article

\begin{abstract}
In this paper, we present a generalized concept of second-order differentiability for interval-valued functions. The global existence of solutions for interval-valued second-order differential equations (ISDEs) under generalized $\mathrm{H}$-differentiability is proved. We present some examples of linear interval-valued second-order differential equations with initial conditions having four different solutions. Finally, we propose a new algorithm based on the analysis of a crisp solution. Some examples are given by using our proposed algorithm.
\end{abstract}

MSC: $34 \mathrm{~K} 05 ; 34 \mathrm{~K} 30 ; 47 \mathrm{G} 20$

Keywords: interval-valued differential equations; interval-valued second-order differential equations; generalized Hukuhara derivative; global existence

\section{Introduction}

The set-valued differential and integral equations are an important part of the theory of set-valued analysis. They have the important value of theory and application in control theory; and they were studied in 1969 by De Blasi and Iervolino [1]. Recently, set-valued differential equations have been studied by many authors due to their application in many areas. For the basic theory on set-valued differential and integral equations, the readers can be referred to the good books and papers [2-10] and references therein. The intervalvalued analysis and interval differential equations (IDEs) are the particular cases of the set-valued analysis and set differential equations, respectively. In many cases, when modeling real-world phenomena, information about the behavior of a dynamic system is uncertain, and we have to consider these uncertainties to gain more models. Interval-valued differential and integro-differential equations are a natural way to model dynamic systems subject to uncertainties. Recently, many works have been done by several authors in the theory of interval-valued differential equations (see, e.g., [11-15]). There are several approaches to the study of interval differential equations. One popular approach is based on $\mathrm{H}$-differentiability. The approach based on $\mathrm{H}$-derivative has the disadvantage that it leads to solutions which have an increasing length of their support. Note that this definition of derivative is very restrictive; for instance, we can see that if $X(t)=A \cdot x(t)$, where $A$ is an interval-valued constant and $x:[a, b] \rightarrow \mathbb{R}_{+}$is a real function with $x^{\prime}(t)<0$, then $X(t)$

\section{6ringer}

(02013 Ngo and Nguyen; licensee Springer. This is an Open Access article distributed under the terms of the Creative Commons Attribution License (http://creativecommons.org/licenses/by/2.0), which permits unrestricted use, distribution, and reproduction in any medium, provided the original work is properly cited. 
is not differentiable. Recently, to avoid this difficulty, Stefanini and Bede [12] solved the above mentioned approach under strongly generalized differentiability of interval-valued functions. In this case, the derivative exists and the solution of an interval-valued differential equation may have decreasing length of the support, but the uniqueness is lost. The paper of Stefanini and Bede was a starting point for the topic of interval-valued differential equations (see [14-16]) and later also for fuzzy differential equations.

The connection between the fuzzy analysis and the interval analysis is very well known (Moore and Lodwick [17]). Interval analysis and fuzzy analysis were introduced as an attempt to handle interval uncertainty that appears in many mathematical or computer models of some deterministic real-world phenomena. In [18] authors considered $n$ thorder fuzzy differential equations with initial value conditions and proved the local existence and uniqueness of solution for nonlinearities satisfying a Lipschitz condition. In $[12,19]$ authors studied the existence of the solutions to interval-valued and fuzzy differential equations involving generalized differentiability. Based on the results in [18], authors studied the local existence and uniqueness of solutions for fuzzy second-order integrodifferential equations with fuzzy kernel under strongly generalized H-differentiability [20]. In [21] $n$ th-order fuzzy differential equations (NFDEs) under generalized differentiability were discussed and the existence and uniqueness theorem of solution of NFDE was proved by using the contraction principle in a Banach space. In [22] authors developed the fuzzy improved Runge-Kutta-Nystrom method for solving a second-order fuzzy differential based on the generalized concept of higher-order fuzzy differentiability. Also, a very important generalization and development related to the subject of the present paper is in the field of fuzzy sets, i.e., fuzzy calculus and fuzzy differential equations under the generalized Hukuhara derivative. Recently, several works, e.g., [13, 23-43], have been done on set-valued differential equations, fuzzy differential equations, and fuzzy integrodifferential equations, fractional fuzzy differential equations, and some methods for solving fuzzy differential equations [44-49].

In $[12,14,15]$ the authors presented interval-valued differential equations under generalized Hukuhara differentiability which were given in the following form:

$$
D_{H}^{1, g} X(t)=F(t, X(t)), \quad X\left(t_{0}\right)=X_{0} \in K_{C}(\mathbb{R}), \quad t \in\left[t_{0}, t_{0}+p\right],
$$

where $D_{H}^{1, g}$ denotes two kinds of derivatives, namely the classical Hukuhara derivative and the second type Hukuhara derivative (generalized Hukuhara differentiability). The existence and uniqueness of a Cauchy problem is then obtained under an assumption that the coefficients satisfy a condition with the Lipschitz constant (see [12]). The proof is based on the application of the Banach fixed point theorem. In [15], under the generalized Lipschitz condition, Malinowski obtained the existence and uniqueness of solutions to both kinds of IDEs.

In this paper, we study four kinds of solutions to ISDEs

$$
\begin{aligned}
& D_{H}^{2, g} X(t)=F\left(t, X(t), D_{H}^{1, g} X(t)\right), \quad X\left(t_{0}\right)=I_{1}, \\
& D_{H}^{1, g} X\left(t_{0}\right)=I_{2} \in K_{C}(\mathbb{R}), \quad t \in\left[t_{0}, T\right] .
\end{aligned}
$$

The different types of solutions to ISDEs are generated by the usage of two different concepts of interval-valued second-order derivative. This direction of research is motivated 
by the results of Stefanini and Bede [12], Malinowski [14, 15] concerning deterministic IDEs with generalized interval-valued derivative.

This paper is organized as follows. In Section 2, we recall some basic concepts and notations about interval analysis and interval-valued differential equations. In Section 3, we present the global existence and uniqueness theorem of a solution to the interval-valued second-order differential equation under two kinds of the Hukuhara derivative. Some examples of linear second-order interval-valued differential equations with initial conditions having four different solutions are presented. In Section 4, we propose a new algorithm based on the analysis of a crisp solution.

\section{Preliminaries}

Let $K_{C}(\mathbb{R})$ denote the family all nonempty, compact and convex subsets of $\mathbb{R}$. The addition and scalar multiplication in $K_{C}(\mathbb{R})$, we define as usual, i.e., for $A, B \in K_{C}(\mathbb{R}), A=[\underline{A}, \bar{A}]$, $B=[\underline{B}, \bar{B}]$, where $\underline{A} \leq \bar{A}, \underline{B} \leq \bar{B}$ and $\lambda \geq 0$, then we have

$$
A+B=[\underline{A}+\underline{B}, \bar{A}+\bar{B}], \quad \lambda A=[\lambda \underline{A}, \lambda \bar{A}] \quad(-\lambda A=[\lambda \bar{A}, \lambda \underline{A}]) .
$$

Furthermore, let $A \in K_{C}(\mathbb{R}), \lambda_{1}, \lambda_{2}, \lambda_{3}, \lambda_{4} \in R$ and $\lambda_{3} \lambda_{4} \geq 0$, then we have $\lambda_{1}\left(\lambda_{2} A\right)=$ $\left(\lambda_{1} \lambda_{2}\right) A$ and $\left(\lambda_{3}+\lambda_{4}\right) A=\lambda_{3} A+\lambda_{4} A$. Let $A, B \in K_{C}(\mathbb{R})$ as above. Then the Hausdorff metric $H$ in $K_{C}(\mathbb{R})$ is defined as follows:

$$
H(A, B)=\max \{|\underline{A}-\underline{B}|,|\bar{A}-\bar{B}|\} .
$$

We notice that $\left(K_{C}(\mathbb{R}), H\right)$ is a complete, separable and locally compact metric space.

We define the magnitude and length of $A \in K_{C}(\mathbb{R})$ by

$$
H(A,\{0\})=\|A\|=\max \{|\underline{A}|,|\bar{A}|\}, \quad \operatorname{len}(A)=\bar{A}-\underline{A},
$$

respectively, where $\{0\}$ is the zero element of $K_{C}(\mathbb{R})$, which is regarded as one point.

The Hausdorff metric (2.1) satisfies the following properties:

$$
\begin{aligned}
& H(A+C, B+C)=H(A, B) \text { and } H(A, B)=H(B, A), \\
& H(A+B, C+D) \leq H(A, C)+H(B, D), \\
& H(\lambda A, \lambda B)=|\lambda| H(A, B), \\
& H(A, B) \leq H(A, C)+H(C, B)
\end{aligned}
$$

for all $A, B, C, D \in K_{C}(\mathbb{R})$ and $\lambda \in R$. Let $A, B \in K_{C}(\mathbb{R})$. If there exists an interval $C \in K_{C}(\mathbb{R})$ such that $A=B+C$, then we call $C$ the Hukuhara difference of $A$ and $B$. We denote the interval $C$ by $A \ominus B$. Note that $A \ominus B \neq A+(-) B$. It is known that $A \ominus B$ exists in the case len $(A) \geq \operatorname{len}(B)$. Besides that, we can see [14] the following properties for $A, B, C, D \in$ $K_{C}(\mathbb{R})$ :

- If $A \ominus B, A \ominus C$ exist, then $H(A \ominus B, A \ominus C)=H(B, C)$;

- If $A \ominus B, C \ominus D$ exist, then $H(A \ominus B, C \ominus D)=H(A+D, B+C)$;

- If $A \ominus B, A \ominus(B+C)$ exist, then there exist $(A \ominus B) \ominus C$ and $(A \ominus B) \ominus C=A \ominus(B+C)$; 
- If $A \ominus B, A \ominus C, C \ominus B$ exist, then there exist $(A \ominus B) \ominus(A \ominus C)$ and $(A \ominus B) \ominus(A \ominus C)=C \ominus B$.

Definition 2.1 We say that the interval-valued mapping $F:[a, b] \subset R^{+} \rightarrow K_{C}(\mathbb{R})$ is continuous at the point $t \in[a, b]$ if for every $\varepsilon>0$ there exists $\delta=\delta(t, \varepsilon)>0$ such that, for all $s \in[a, b]$ such that $|t-s|<\delta$, one has $H(F(t), F(s)) \leq \varepsilon$.

The strongly generalized differentiability was introduced in [12] and studied in $[14,38-$ $40]$.

Definition 2.2 Let $X:[a, b] \rightarrow K_{C}(\mathbb{R})$ and $t \in[a, b]$. We say that $X$ is strongly generalized differentiable of the first-order differential at $t$ if there exists $D_{H}^{1, g} X(t) \in K_{C}(\mathbb{R})$ such that

(i) for all $h>0$ sufficiently small, $\exists X(t+h) \ominus X(t), \exists X(t) \ominus X(t-h)$ and

$$
\begin{aligned}
& \lim _{h \searrow 0} H\left(\frac{X(t+h) \ominus X(t)}{h}, D_{H}^{1, g} X(t)\right)=0, \\
& \lim _{h \searrow 0} H\left(\frac{X(t) \ominus X(t-h)}{h}, D_{H}^{1, g} X(t)\right)=0,
\end{aligned}
$$

or

(ii) for all $h>0$ sufficiently small, $\exists X(t) \ominus X(t+h), \exists X(t-h) \ominus X(t)$ and

$$
\begin{aligned}
& \lim _{h \searrow 0} H\left(\frac{X(t) \ominus X(t+h)}{-h}, D_{H}^{1, g} X(t)\right)=0, \\
& \lim _{h \searrow 0} H\left(\frac{X(t-h) \ominus X(t)}{-h}, D_{H}^{1, g} X(t)\right)=0,
\end{aligned}
$$

or

(iii) for all $h>0$ sufficiently small, $\exists X(t+h) \ominus X(t), \exists X(t-h) \ominus X(t)$ and

$$
\begin{aligned}
& \lim _{h \searrow 0} H\left(\frac{X(t+h) \ominus X(t)}{h}, D_{H}^{1, g} X(t)\right)=0, \\
& \lim _{h \searrow 0} H\left(\frac{X(t-h) \ominus X(t)}{-h}, D_{H}^{1, g} X(t)\right)=0,
\end{aligned}
$$

or

(iv) for all $h>0$ sufficiently small, $\exists X(t) \ominus X(t+h), \exists X(t) \ominus X(t-h)$ and the limits

$$
\begin{aligned}
& \lim _{h \searrow 0} H\left(\frac{X(t) \ominus X(t+h)}{-h}, D_{H}^{1, g} X(t)\right)=0, \\
& \lim _{h \searrow 0} H\left(\frac{X(t) \ominus X(t-h)}{h}, D_{H}^{1, g} X(t)\right)=0
\end{aligned}
$$

( $h$ at denominators means $\frac{1}{h}$ ). In this definition, case (i) ((i)-differentiability for short) corresponds to the classical $\mathrm{H}$-derivative, so this differentiability concept is a generalization of the Hukuhara derivative.

Lemma $2.1[12,14,15]$ The interval-valued differential equation $D_{H}^{1, g} X(t)=F(t, X(t))$, $X(a)=X_{0} \in K_{C}(\mathbb{R})$, where $F:[a, b] \times K_{C}(\mathbb{R}) \rightarrow K_{C}(\mathbb{R})$ is supposed to be continuous, is 
equivalent to one of the integral equations

$$
\begin{aligned}
& X(t)=X_{0}+\int_{a}^{t} F(s, X(s)) d s, \quad \forall t \in[a, b] \quad \text { or } \\
& X(a)=X(t)+(-1) \cdot \int_{a}^{t} F(s, X(s)) d s, \quad \forall t \in[a, b]
\end{aligned}
$$

on the interval $[a, b] \in R$, under the strong differentiability condition, (i) or (ii), respectively. We notice that the equivalence between two equations in this lemma means that any solution is a solution for the other one.

Definition 2.3 Let $X:[a, b] \rightarrow K_{C}(\mathbb{R})$ and $t \in[a, b]$. We say that $X$ is strongly generalized differentiable of the second-order differential at $t$ if there exists $D_{H}^{2, g} X(t) \in K_{C}(\mathbb{R})$ such that

(i) for all $h>0$ sufficiently small, $\exists D_{H}^{1, g} X(t+h) \ominus D_{H}^{1, g} X(t), \exists D_{H}^{1, g} X(t) \ominus D_{H}^{1, g} X(t-h)$ and the following limits hold (in the metric $H$ )

$$
\lim _{h \searrow 0} \frac{D_{H}^{1, g} X(t+h) \ominus D_{H}^{1, g} X(t)}{h}=\lim _{h \searrow 0} \frac{D_{H}^{1, g} X(t) \ominus D_{H}^{1, g} X(t-h)}{h}=D_{H}^{2, g} X(t),
$$

or

(ii) for all $h>0$ sufficiently small, $\exists D_{H}^{1, g} X(t) \ominus D_{H}^{1, g} X(t+h), \exists D_{H}^{1, g} X(t-h) \ominus D_{H}^{1, g} X(t)$ and the following limits hold (in the metric $H$ )

$$
\lim _{h \searrow 0} \frac{D_{H}^{1, g} X(t) \ominus D_{H}^{1, g} X(t+h)}{-h}=\lim _{h \searrow 0} \frac{D_{H}^{1, g} X(t-h) \ominus D_{H}^{1, g} X(t)}{-h}=D_{H}^{2, g} X(t),
$$

or

(iii) for all $h>0$ sufficiently small, $\exists D_{H}^{1, g} X(t+h) \ominus D_{H}^{1, g} X(t), \exists D_{H}^{1, g} X(t-h) \ominus D_{H}^{1, g} X(t)$ and the following limits hold (in the metric $H$ )

$$
\lim _{h \searrow 0} \frac{D_{H}^{1, g} X(t+h) \ominus D_{H}^{1, g} X(t)}{h}=\lim _{h \searrow 0} \frac{D_{H}^{1, g} X(t-h) \ominus D_{H}^{1, g} X(t)}{-h}=D_{H}^{2, g} X(t),
$$

or

(iv) for all $h>0$ sufficiently small, $\exists D_{H}^{1, g} X(t) \ominus D_{H}^{1, g} X(t+h), \exists D_{H}^{1, g} X(t) \ominus D_{H}^{1, g} X(t-h)$ and the following limits hold (in the metric $H$ )

$$
\lim _{h \searrow 0} \frac{D_{H}^{1, g} X(t) \ominus D_{H}^{1, g} X(t+h)}{-h}=\lim _{h \searrow 0} \frac{D_{H}^{1, g} X(t) \ominus D_{H}^{1, g} X(t-h)}{h}=D_{H}^{2, g} X(t) .
$$

In this paper we consider only the two first items of Definition 2.3. In the other cases, the derivative is trivial because it is reduced to a crisp element. Let us consider the intervalvalued second differential equation initial value problem (ISDE) of the form

$$
D_{H}^{2, g} X(t)=F\left(t, X(t), D_{H}^{1, g} X(t)\right), \quad X\left(t_{0}\right)=I_{1}, \quad D_{H}^{2, g} X\left(t_{0}\right)=I_{2}, \quad t \in\left[t_{0}, T\right],
$$

where $X(t)=[\underline{X}(t), \bar{X}(t)]$ is an interval-valued function of $t, F\left(t, X(t), D_{H}^{1, g} X(t)\right)$ is an interval-valued function and the interval-valued variables $D_{H}^{1, g} X(t), D_{H}^{2, g} X(t)$ are defined as the derivatives of $X(t)$ and $D_{H}^{1, g} X(t)$, respectively. 
Theorem 2.1 Let $X:\left[t_{0}, T\right] \rightarrow K_{C}(\mathbb{R})$ and $D_{H}^{1, g} X:\left[t_{0}, T\right] \rightarrow K_{C}(\mathbb{R})$ be interval-valued functions, where $X(t)=[\underline{X}(t), \bar{X}(t)]$. If $X, D_{H}^{1, g} X$ are (i)-differentiable or (ii)-differentiable, then $\underline{X}(t), \bar{X}(t)$ and $(\underline{X}(t))^{\prime},(\bar{X}(t))^{\prime}$ are differentiable functions and

(i) $D_{H}^{2, g} X(t)=\left[(\underline{X}(t))^{\prime \prime},(\bar{X}(t))^{\prime \prime}\right]$, where $X$ and $D_{H}^{1, g} X$ are (i)-differentiable;

(ii) $D_{H}^{2, g} X(t)=\left[(\bar{X}(t))^{\prime \prime},(\underline{X}(t))^{\prime \prime}\right]$, where $X$ is (i)-differentiable and $D_{H}^{1, g} X$ is

(ii)-differentiable;

(iii) $D_{H}^{2, g} X(t)=\left[(\bar{X}(t))^{\prime \prime},(\underline{X}(t))^{\prime \prime}\right]$, where $X$ is (ii)-differentiable and $D_{H}^{1, g} X$ is

(i)-differentiable;

(iv) $D_{H}^{2, g} X(t)=\left[(\underline{X}(t))^{\prime \prime},(\bar{X}(t))^{\prime \prime}\right]$, where $X$ and $D_{H}^{1, g} X$ are (i)-differentiable.

Definition 2.4 Let $X:\left[t_{0}, T\right] \rightarrow K_{C}(\mathbb{R})$ and $D_{H}^{1, g} X:\left[t_{0}, T\right] \rightarrow K_{C}(\mathbb{R})$, we define:

(a) The space of continuous functions $X$ by $C\left(\left[t_{0}, T\right], K_{C}(\mathbb{R})\right)$ with the distance

$$
H_{0}(X, Y)=\sup _{t \in\left[t_{0}, T\right]}\{H(X, Y) \exp (-k t)\}
$$

(b) The space of continuous functions $D_{H}^{1, g} X(t)$ by $C^{1}\left(\left[t_{0}, T\right], K_{C}(\mathbb{R})\right)$ with the distance

$$
H_{0}^{1}(X, Y)=H_{0}(X, Y)+H_{0}\left(D_{H}^{1, g} X, D_{H}^{1, g} Y\right)
$$

where we assume that $D_{H}^{1, g} X, D_{H}^{1, g} Y$ exist and $k \in \mathbb{R}_{+}$.

In this definition, we know that the space $\left(C\left(\left[t_{0}, T\right], K_{C}(\mathbb{R})\right), H_{0}\right)$ of continuous functions $X:\left[t_{0}, T\right] \rightarrow K_{C}(\mathbb{R})$ is a complete metric space with distance (2.3).

Lemma $2.2\left(C^{1}\left(\left[t_{0}, T\right], K_{C}(\mathbb{R})\right), H_{0}^{1}\right)$ is a complete metric space.

Based on Definition 2.3, there are two possible cases for each derivation, so there are four possible cases for the second-order derivation.

Theorem 2.2 Assume that $F:\left[t_{0}, T\right] \times K_{C}(\mathbb{R}) \times K_{C}(\mathbb{R}) \rightarrow K_{C}(\mathbb{R})$ is continuous. A mapping $X:\left[t_{0}, T\right] \rightarrow K_{C C}(R)$ is a solution to problem (2.2) if and only if $X$ and $D_{H}^{1, g} X(t)$ are continuous on $\left[t_{0}, T\right]$ and satisfy one of the following interval integral equations:

(i) $X(t)=I_{1}+I_{2}\left(t-t_{0}\right)+\int_{t_{0}}^{t}\left(\int_{t_{0}}^{t} F\left(s, X(s), D_{H}^{1, g} X(s)\right) d s\right) d s$, where $D_{H}^{1, g} X(t)$ and $D_{H}^{2, g} X(t)$ are (i)-differentials, or

(ii) $X(t)=I_{1} \ominus(-1)\left(I_{2}\left(t-t_{0}\right)+\int_{t_{0}}^{t}\left(\int_{t_{0}}^{t} F\left(s, X(s), D_{H}^{1, g} X(s)\right) d s\right) d s\right)$, where $D_{H}^{1, g} X(t)$ is (i)-differential and $D_{H}^{2, g} X(t)$ is (ii)-differential, or

(iii) $X(t)=I_{1}+I_{2}\left(t-t_{0}\right) \ominus(-1) \int_{t_{0}}^{t}\left(\int_{t_{0}}^{t} F\left(s, X(s), D_{H}^{1, g} X(s)\right) d s\right) d s$, where $D_{H}^{1, g} X(t)$ is (ii)-differential and $D_{H}^{2, g} X(t)$ is (i)-differential, or

(iv) $X(t)=I_{1} \ominus\left(I_{2}\left(t-t_{0}\right) \ominus(-1) \int_{t_{0}}^{t}\left(\int_{t_{0}}^{t} F\left(s, X(s), D_{H}^{1, g} X(s)\right) d s\right) d s\right)$, where $D_{H}^{1, g} X(t)$ and $D_{H}^{2, g} X(t)$ are (ii)-differentials.

Definition 2.5 Let $X:\left[t_{0}, T\right] \rightarrow K_{C}(\mathbb{R}), D_{H}^{1, g} X:\left[t_{0}, T\right] \rightarrow K_{C}(\mathbb{R})$ be interval-valued functions which are (i)-differentiable. If $X, D_{H}^{1, g} X$ and their derivatives satisfy problem (2.2), we say $X$ is a (i-i)-solution of problem (2.2).

Definition 2.6 Let $X:\left[t_{0}, T\right] \rightarrow K_{C}(\mathbb{R}), D_{H}^{1, g} X:\left[t_{0}, T\right] \rightarrow K_{C}(\mathbb{R})$ be interval-valued functions which are (i)-differentiable and (ii)-differentiable, respectively. If $X, D_{H}^{1, g} X$ and their derivatives satisfy problem (2.2), we say $X$ is a (i-ii)-solution of problem (2.2). 
Definition 2.7 Let $X:\left[t_{0}, T\right] \rightarrow K_{C}(\mathbb{R}), D_{H}^{1, g} X:\left[t_{0}, T\right] \rightarrow K_{C}(\mathbb{R})$ be interval-valued functions which are (ii)-differentiable and (i)-differentiable, respectively. If $X, D_{H}^{1, g} X$ and their derivatives satisfy problem (2.2), we say $X$ is a (ii-i)-solution of problem (2.2).

Definition 2.8 Let $X:\left[t_{0}, T\right] \rightarrow K_{C}(\mathbb{R}), D_{H}^{1, g} X:\left[t_{0}, T\right] \rightarrow K_{C}(\mathbb{R})$ be interval-valued functions which are (ii)-differentiable. If $X, D_{H}^{1, g} X$ and their derivatives satisfy problem (2.2), we say $X$ is a (ii-ii)-solution of problem (2.2).

Theorem 2.3 Let $F:\left[t_{0}, T\right] \times K_{C}(\mathbb{R}) \times K_{C}(\mathbb{R}) \rightarrow K_{C}(\mathbb{R})$ be continuous, and suppose that there exist $L_{1}, L_{2} \in \mathbb{R}+$ such that

$$
H\left(F\left(t, X_{1}, Y_{1}\right), F\left(t, X_{2}, Y_{2}\right)\right) \leq L_{1} H\left(X_{1}, X_{2}\right)+L_{2} H\left(Y_{1}, Y_{2}\right)
$$

for all $t \in\left[t_{0}, T\right], X_{1}, X_{2}, Y_{1}, Y_{2} \in K_{C}(\mathbb{R})$. Then problem (2.2) has a unique local solution on some intervals $\left[t_{0}, \mathbb{T}\right]\left(\mathbb{T} \leq T-t_{0}\right)$ for each case.

\section{Global existence of solutions for interval-valued second-order differential equations}

In this section of the paper, we shall consider again the following initial value problem for the interval-valued second-order differential equation (ISDE) of the form

$$
D_{H}^{2, g} X(t)=F\left(t, X(t), D_{H}^{1, g} X(t)\right), \quad X\left(t_{0}\right)=I_{1}, \quad D_{H}^{1, g} X\left(t_{0}\right)=I_{2}
$$

for all $t \in I=\left[t_{0}, \infty\right)$, where $F: I \times K_{C}(\mathbb{R}) \times K_{C}(\mathbb{R}) \rightarrow K_{C}(\mathbb{R})$.

\section{Theorem 3.1 Assume that}

(C1) $F(\cdot, \cdot, \cdot): I \times K_{C}(\mathbb{R}) \times K_{C}(\mathbb{R}) \rightarrow K_{C}(\mathbb{R})$ is locally Lipschitzian in $X, D_{H}^{1, g} X(t)$;

(C2) $H\left(F\left(t, X, D_{H}^{1, g} X(t)\right),\{0\}\right) \leq g\left(t, H(X,\{0\}), H\left(D_{H}^{1, g} X(t),\{0\}\right)\right)$, for all $\left(t, X, D_{H}^{1, g} X(t)\right) \in I \times K_{C}(\mathbb{R}) \times K_{C}(\mathbb{R})$, where $g \in C\left[I \times \mathbb{R}^{+} \times \mathbb{R}^{+}, \mathbb{R}^{+}\right]$is a nondecreasing function for each $t \in I$, and the maximal solution $r\left(t, t_{0}, u_{0}, u_{0}^{\prime}\right)$ of the scalar initial value problem

$$
\frac{d^{2}}{d t} u(t)=g\left(t, u, u^{\prime}\right), \quad u\left(t_{0}\right)=u_{0}, \quad u^{\prime}\left(t_{0}\right)=u_{0}^{\prime},
$$

exists throughout I;

(C3) $H\left(X\left(t, t_{0}, X_{0}, D_{H}^{1, g} X\left(t_{0}\right)\right),\{0\}\right) \leq r\left(t, t_{0}, u_{0}, u_{0}^{\prime}\right), H\left(X_{0},\{0\}\right) \leq u_{0}$ and $H\left(D_{H}^{1, g} X\left(t_{0}\right),\{0\}\right) \leq u_{0}^{\prime}$.

Then the largest interval of the existence of any solution $X\left(t, t_{0}, X_{0}, D_{H}^{1, g} X\left(t_{0}\right)\right)$ of (3.1) for each case is I. In addition, if $r\left(t, t_{0}, u_{0}, u_{0}^{\prime}\right)$ is bounded on $I$, then $\lim _{t \rightarrow \infty} X\left(t, t_{0}, X_{0}, D_{H}^{1, g} X\left(t_{0}\right)\right)$ exists.

Proof Since the way of the proof is similar for four cases, we only prove the case $X$ and $D_{H}^{1, g} X$ are (i)-differentiable. By hypothesis $(\mathrm{C} 1)$, there exists $T>t_{0}$ such that the unique solution of problem (3.1) exists on $I$. Let $\mathcal{S}=\left\{X(t) \mid X(t)\right.$ is defined on $\left[t_{0}, \beta_{X}\right]$ and is the solution to (3.1)\}. Then $\mathcal{S} \neq \emptyset$. Taking $\beta=\sup \left\{\beta_{X} \mid X(t) \in \mathcal{S}\right\}$, clearly, there exists a unique solution of (3.1) which is defined on $\left[t_{0}, \beta\right)$ with $H\left(X_{0},\{0\}\right) \leq u_{0}$ and $H\left(D_{H}^{1, g} X\left(t_{0}\right),\{0\}\right) \leq u_{0}^{\prime}$. 
If $\beta=\infty$, obviously, the theorem is proved. Hence we suppose $\beta<\infty$ and define $m(t)=$ $H\left(X\left(t, t_{0}, X_{0}, D_{H}^{1, g} X\left(t_{0}\right)\right),\{0\}\right), t_{0} \leq t<\beta$. Then we have

$$
\begin{aligned}
D_{+} m^{\prime}(t)= & \lim _{h \rightarrow 0^{+}} \inf \left(\frac{H\left(D_{H}^{1, g} X\left(t+h, t_{0}, X_{0}, D_{H}^{1, g} X\left(t_{0}\right)\right),\{0\}\right)}{h}\right. \\
& \left.-\frac{H\left(D_{H}^{1, g} X\left(t, t_{0}, X_{0}, D_{H}^{1, g} X\left(t_{0}\right)\right),\{0\}\right)}{h}\right) \\
\leq & \lim _{h \rightarrow 0^{+}} \inf \frac{H\left(D_{H}^{1, g} X\left(t+h, t_{0}, X_{0}, D_{H}^{1, g} X\left(t_{0}\right)\right), D_{H}^{1, g} X\left(t, t_{0}, X_{0}, D_{H}^{1, g} X\left(t_{0}\right)\right)\right)}{h} \\
= & \lim _{h \rightarrow 0^{+}} \inf H\left(\frac{D_{H}^{1, g} X\left(t+h, t_{0}, X_{0}, D_{H}^{1, g} X\left(t_{0}\right)\right) \ominus D_{H}^{1, g} X\left(t, t_{0}, X_{0}, D_{H}^{1, g} X\left(t_{0}\right)\right)}{h},\{0\}\right) \\
= & H\left(F\left(t, X\left(t, t_{0}, X_{0}, D_{H}^{1, g} X\left(t_{0}\right)\right), D_{H}^{1, g} X\left(t, t_{0}, X_{0}, D_{H}^{1, g} X\left(t_{0}\right)\right)\right),\{0\}\right) \\
\leq & g\left(t, m(t), m^{\prime}(t)\right)
\end{aligned}
$$

for all $t_{0} \leq t<\beta$ and $m\left(t_{0}\right)=H\left(X_{0},\{0\}\right) \leq u_{0}$. Further, by assumption (C3), it follows that $m(t) \leq r\left(t, t_{0}, u_{0}, u_{0}^{\prime}\right)$. Now, we show that $\lim _{t \rightarrow \infty} X\left(t, t_{0}, X_{0}, D_{H}^{1, g} X\left(t_{0}\right)\right)$ exists. In fact, for any $t_{1}, t_{2}$ such that $t_{0} \leq t_{1}<t_{2}<\beta$, we have

$$
\begin{aligned}
H( & \left.X\left(t_{1}, t_{0}, X_{0}, D_{H}^{1, g} X\left(t_{0}\right)\right), X\left(t_{2}, t_{0}, X_{0}, D_{H}^{1, g} X\left(t_{0}\right)\right)\right) \\
= & H\left(\begin{array}{c}
I_{1}+\left(I_{2}\left(t_{1}-t_{0}\right)+\int_{t_{0}}^{t_{1}}\left(\int_{t_{0}}^{s} F\left(\tau, X(\tau), D_{H}^{1, g} X(\tau)\right) d \tau\right) d s\right), \\
I_{1}+\left(I_{2}\left(t_{2}-t_{0}\right)+\int_{t_{0}}^{t_{2}}\left(\int_{t_{0}}^{s} F\left(\tau, X(\tau), D_{H}^{1, g} X(\tau)\right) d \tau\right) d s\right)
\end{array}\right) \\
\leq & H\left(\int_{t_{0}}^{t_{1}}\left(\int_{t_{0}}^{s} F\left(\tau, X(\tau), D_{H}^{1, g} X(\tau)\right) d \tau\right) d s,\right. \\
& \left.\int_{t_{0}}^{t_{2}}\left(\int_{t_{0}}^{s} F\left(\tau, X(\tau), D_{H}^{1, g} X(\tau)\right) d \tau\right) d s\right) \\
\leq & \int_{t_{1}}^{t_{2}}\left(\int_{t_{0}}^{s} H\left(F\left(\tau, X(\tau), D_{H}^{1, g} X(\tau)\right),\{0\}\right) d \tau\right) d s \\
\leq & r\left(t_{2}, t_{0}, u_{0}, u_{0}^{\prime}\right)-r\left(t_{1}, t_{0}, u_{0}, u_{0}^{\prime}\right) .
\end{aligned}
$$

Since $\lim _{t \rightarrow \beta-0} r(t)$ exists and is finite, taking the limits as $t_{1}, t_{2} \rightarrow \beta-0$ and using the completeness of $\left(K_{C}(\mathbb{R}), H\right)$ in the last inequality, we see that $\lim _{t \rightarrow \beta-0} X\left(t, t_{0}, X_{0}, D_{H}^{1, g} X\left(t_{0}\right)\right)$ exists in $\left(K_{C}(\mathbb{R}), H\right)$. Define $X(\beta)=\lim _{t \rightarrow \beta-0} X\left(t, t_{0}, X_{0}, D_{H}^{1, g} X\left(t_{0}\right)\right)$ and consider the IVP

$$
D_{H}^{2, g} X(t)=F\left(t, X, D_{H}^{1, g} X(t)\right), \quad X(\beta)=\lim _{t \rightarrow \beta-0} X(t) .
$$

Therefore $X(t)$ can be extended beyond $\beta$, which contradicts our assumption. In addition, since $r\left(t, t_{0}, u_{0}, u_{0}^{\prime}\right)$ is bounded and nondecreasing on $I$, it follows that $\lim _{t \rightarrow \infty} X\left(t, t_{0}, X_{0}\right.$, $\left.D_{H}^{1, g} X\left(t_{0}\right)\right)$ exists and is finite.

Example 3.2 Consider the linear second-order IDE

$$
D_{H}^{2, g} X(t)=A(t) D_{H}^{1, g} X(t)+B(t) X(t), \quad X\left(t_{0}\right)=I_{1}, \quad D_{H}^{1, g} X\left(t_{0}\right)=I_{2},
$$

where we assume that $A(\cdot), B(\cdot): \mathbb{R}^{+} \rightarrow \mathbb{R}^{+}$are continuous functions, then the solutions of (3.3) for each case are on $\left[t_{0}, \infty\right)$. 
We see that $F\left(t, X, D_{H}^{1, g} X(t)\right)=A(t) D_{H}^{1, g} X(t)+B(t) X(t)$ is locally Lipschitzian. If we let $g\left(t, u, u^{\prime}\right)=A(t) u^{\prime}+B(t) u$, then $u(t) \equiv 0$ is a unique solution of second-order initial differential equation as follows:

$$
u^{\prime \prime}(t)=A(t) u^{\prime}(t)+B(t) u(t), \quad u\left(t_{0}\right)=0, \quad u^{\prime}\left(t_{0}\right)=0
$$

on $\left[t_{0}, \infty\right)$. In the other hand, we have

$$
\begin{aligned}
H\left(F\left(t, X, D_{H}^{1, g} X(t)\right),\{0\}\right) & =H\left(A(t) D_{H}^{1, g} X(t)+B(t) X(t),\{0\}\right) \\
& \leq B(t) H(X,\{0\})+A(t) H\left(D_{H}^{1, g} X(t),\{0\}\right) \\
& =g\left(t, H(X,\{0\}), H\left(D_{H}^{1, g} X(t),\{0\}\right)\right) .
\end{aligned}
$$

Therefore, the solutions of Example 3.2 are on $\left[t_{0}, \infty\right)$.

\section{Theorem 3.3 Assume that}

(C4) $F \in C\left[I \times K_{C}(\mathbb{R}) \times K_{C}(\mathbb{R})\right], F$ is bounded on bounded sets, and there exists a local solution of problem (3.1) for every $\left(t_{0}, X_{0}, D_{H}^{1, g} X\left(t_{0}\right)\right) \in I \times K_{C}(\mathbb{R}) \times K_{C}(\mathbb{R})$;

(C5) $V \in C\left[I \times K_{C}(\mathbb{R}) \times K_{C}(\mathbb{R}), \mathbb{R}^{+}\right], V$ is locally Lipschitzian in $X, D_{H}^{1, g} X(t)$, $V\left(t, X, D_{H}^{1, g} X(t)\right) \rightarrow \infty$ as $\max \left\{H(X,\{0\}), H\left(D_{H}^{1, g} X(t),\{0\}\right)\right\} \rightarrow \infty$ uniformly for $t \in I$. In addition,

$$
\begin{aligned}
\lim _{h \rightarrow 0^{+}} \inf & \frac{1}{h}\left[V \left(t+h, X+h \int_{t_{0}}^{t} F\left(s, X, D_{H}^{1, g} X(t)\right) d s,\right.\right. \\
& \left.\left.D_{H}^{1, g} X(t)+h F\left(t, X, D_{H}^{1, g} X(t)\right)\right)-V\left(t, X, D_{H}^{1, g} X(t)\right)\right] \\
\leq & g\left(t, V(t, X), V^{\prime}(t, X)\right),
\end{aligned}
$$

where $g \in C\left[I \times \mathbb{R}^{+} \times \mathbb{R}^{+}, \mathbb{R}\right]$;

(C6) the maximal solution $r\left(t, t_{0}, u_{0}, u_{0}^{\prime}\right)$ of problem (3.2) exists on $\left[t_{0}, \infty\right)$ and is positive whenever $u_{0}, u_{0}^{\prime}>0$.

Then, for every $X_{0}, D_{H}^{1, g} X\left(t_{0}\right) \in K_{C}(\mathbb{R})$ such that $V\left(t_{0}, X_{0}, D_{H}^{1, g} X\left(t_{0}\right)\right) \leq \max \left\{u_{0}, u_{0}^{\prime}\right\}$, problem $(3.1)$ has a (i-i)-solution $X(t)$ on $\left[t_{0}, \infty\right)$, which satisfies the estimate $V\left(t, X, D_{H}^{1, g} X(t)\right) \leq$ $r\left(t, t_{0}, u_{0}, u_{0}^{\prime}\right), t \geq t_{0}$.

Proof Let $\mathbb{S}$ denote the set of all functions $X$ defined on $I_{X}=\left[t_{0}, c_{X}\right)$ with values in $K_{C}(\mathbb{R})$ such that $X(t)$ is a (i-i)-solution of problem (3.1) on $I_{X}$ and $V\left(t, X(t), D_{H}^{1, g} X(t)\right) \leq$ $r\left(t, t_{0}, u_{0}, u_{0}^{\prime}\right), t \in I_{X}$. We define a partial order $\leq$ on $\mathbb{S}$ as follows: the relation $X \leq Y$ implies that $I_{X} \subseteq I_{Y}$ and $Y(t) \equiv X(t)$ on $I_{X}$. We shall first show that $\mathbb{S}$ is nonempty. Indeed, by assumption (C4), there exists a (i-i)-solution $X(t)$ of problem (3.1) defined on $I_{X}=\left[t_{0}, c_{X}\right)$. Let $X(t)=X\left(t, t_{0}, X_{0}, D_{H}^{1, g} X\left(t_{0}\right)\right)$ be any (i-i)-solution of (3.1) existing on $I_{X}$. Define $k(t)=$ $V\left(t, X(t), D_{H}^{1, g} X(t)\right)$ so that $k\left(t_{0}\right)=V\left(t_{0}, X_{0}, D_{H}^{1, g} X\left(t_{0}\right)\right) \leq \max \left\{u_{0}, u_{0}^{\prime}\right\}$. Now, for small $h>0$ and using assumption (C5), we consider

$$
\begin{aligned}
k(t+h)-k(t) & =V\left(t+h, X(t+h), D_{H}^{1, g} X(t+h)\right)-V\left(t, X(t), D_{H}^{1, g} X(t)\right) \\
& \leq V\left(t+h, X(t+h), D_{H}^{1, g} X(t+h)\right)
\end{aligned}
$$




$$
\begin{aligned}
& +V\left(t+h, X+h \int_{t_{0}}^{t} F\left(s, X, D_{H}^{1, g} X(t)\right) d s, D_{H}^{1, g} X(t)+h F\left(t, X, D_{H}^{1, g} X(t)\right)\right) \\
& -V\left(t+h, X+h \int_{t_{0}}^{t} F\left(s, X, D_{H}^{1, g} X(t)\right) d s, D_{H}^{1, g} X(t)+h F\left(t, X, D_{H}^{1, g} X(t)\right)\right) \\
& -V\left(t, X(t), D_{H}^{1, g} X(t)\right) \\
\leq & L H\left(X(t+h), X(t)+h \int_{t_{0}}^{t} F\left(s, X, D_{H}^{1, g} X(t)\right) d s\right) \\
& +L H\left(D_{H}^{1, g} X(t+h), D_{H}^{1, g} X(t)+h F\left(t, X, D_{H}^{1, g} X(t)\right)\right) \\
& +V\left(t+h, X+h \int_{t_{0}}^{t} F\left(s, X, D_{H}^{1, g} X(t)\right) d s, D_{H}^{1, g} X(t)+h F\left(t, X, D_{H}^{1, g} X(t)\right)\right) \\
& -V\left(t, X(t), D_{H}^{1, g} X(t)\right)
\end{aligned}
$$

using the Lipschitz condition in assumption (C5). Thus

$$
\begin{aligned}
D_{+} k(t) \equiv & \lim _{h \rightarrow 0^{+}} \inf \frac{1}{h}[k(t+h)-k(t)] \\
\leq & D_{+} V\left(t, X(t), D_{H}^{1, g} X(t)\right) \\
& +L \lim _{h \rightarrow 0^{+}} \inf \frac{1}{h} H\left(X(t+h), X(t)+h \int_{t_{0}}^{t} F\left(s, X, D_{H}^{1, g} X(t)\right) d s\right) \\
& +L \lim _{h \rightarrow 0^{+}} \inf \frac{1}{h} H\left(D_{H}^{1, g} X(t+h), D_{H}^{1, g} X(t)+h F\left(t, X, D_{H}^{1, g} X(t)\right)\right) .
\end{aligned}
$$

Since

$$
\begin{gathered}
\lim _{h \rightarrow 0^{+}} \inf \frac{1}{h} H\left(X(t+h), X(t)+h \int_{t_{0}}^{t} F\left(s, X, D_{H}^{1, g} X(t)\right) d s\right) \\
\quad=H\left(\frac{X(t) \ominus X(t+h)}{-h}, \int_{t_{0}}^{t} F\left(s, X, D_{H}^{1, g} X(t)\right) d s\right)
\end{gathered}
$$

and $X(t)$ is any (i-i)-solution of (3.1), we find that

$$
\begin{aligned}
& \lim _{h \rightarrow 0^{+}} \inf \frac{1}{h} H\left(X(t+h), X(t)+h \int_{t_{0}}^{t} F\left(s, X, D_{H}^{1, g} X(t)\right) d s\right) \\
& =\lim _{h \rightarrow 0^{+}} \inf H\left(\frac{X(t) \ominus X(t+h)}{-h}, \int_{t_{0}}^{t} F\left(s, X, D_{H}^{1, g} X(t)\right) d s\right) \\
& =H\left(D_{H}^{g} X(t), \int_{t_{0}}^{t} F\left(s, X, D_{H}^{1, g} X(t)\right) d s\right)=0 .
\end{aligned}
$$

On the other hand,

$$
\begin{aligned}
& \lim _{h \rightarrow 0^{+}} \inf \frac{1}{h} H\left(D_{H}^{1, g} X(t+h), D_{H}^{1, g} X(t)+h F\left(t, X, D_{H}^{1, g} X(t)\right)\right) \\
& \quad=\lim _{h \rightarrow 0^{+}} \inf H\left(\frac{D_{H}^{1, g} X(t) \ominus D_{H}^{1, g} X(t+h)}{-h}, F\left(t, X, D_{H}^{1, g} X(t)\right)\right) \\
& \quad=H\left(D_{H}^{2, g} X(t), F\left(t, X, D_{H}^{1, g} X(t)\right)\right)=0 .
\end{aligned}
$$


Therefore, we have the scalar differential inequality

$$
D_{+} k(t) \leq g\left(t, k(t), k^{\prime}(t)\right), \quad t \in I_{X} .
$$

We get the estimate

$$
k(t) \leq r\left(t, t_{0}, u_{0}, u_{0}^{\prime}\right), \quad t \in I_{X} .
$$

It follows that

$$
V\left(t, X(t), D_{H}^{1, g} X(t)\right) \leq r\left(t, t_{0}, u_{0}, u_{0}^{\prime}\right), \quad t \in I_{X},
$$

where $r(t)$ is the maximal solution of (3.2). This shows that $X \in \mathbb{S}$, and so $\mathbb{S}$ is nonempty. If $\left(X_{\beta}\right)_{\beta}$ is a chain $(\mathbb{S}, \leq)$, then there is a uniquely defined mapping $Y$ on $J_{Y}=\left[t_{0}, \sup _{\beta} c_{X_{\beta}}\right]$ that coincides with $X_{\beta}$ on $I_{X_{\beta}}$. Clearly, $Y \in \mathbb{S}$ and therefore $Y$ is an upper bound of $\left(X_{\beta}\right)_{\beta}$ in $(\mathbb{S}, \leq)$. The proof of the theorem is completed if we show that $c_{Z}=\infty$. Suppose that it is not true, so that $c_{Z}<\infty$. Since $r(t)$ is assumed to exist on $\left[t_{0}, \infty\right), r(t)$ is bounded on $I_{Z}$. Since $V\left(t, X(t), D_{H}^{1, g} X(t)\right) \rightarrow \infty$ as $H(X(t),\{0\}) \rightarrow \infty$ uniformly in $t$ on $\left[t_{0}, c_{Z}\right]$, the relation $V\left(t, X(t), D_{H}^{1, g} X(t)\right) \leq r(t)$ on $I_{Z}$ implies that $H(Z(t),\{0\})$ is bounded on $I_{Z}$. By assumption (C4), this shows that there is an $M>0$ such that

$$
H\left(F\left(t, X(t), D_{H}^{1, g} X(t)\right),\{0\}\right) \leq M, \quad t \in I_{Z} .
$$

We have, for all $t_{1}, t_{2} \in I_{Z}$ with $t_{1} \leq t_{2}$,

$$
\begin{aligned}
H\left(Z\left(t_{2}\right), Z\left(t_{1}\right)\right) & =H\left(\begin{array}{c}
I_{1}+\left(I_{2}\left(t_{2}-t_{0}\right)+\int_{t_{0}}^{t_{2}}\left(\int_{t_{0}}^{s} F\left(\tau, Z(\tau), D_{H}^{1, g} Z(\tau)\right) d \tau\right) d s\right), \\
I_{1}+\left(I_{2}\left(t_{1}-t_{0}\right)+\int_{t_{0}}^{t_{1}}\left(\int_{t_{0}}^{s} F\left(\tau, Z(\tau), D_{H}^{1, g} Z(\tau)\right) d \tau\right) d s\right)
\end{array}\right) \\
& \leq \int_{t_{1}}^{t_{2}} \int_{t_{0}}^{s} H\left(F\left(\tau, Z(\tau), D_{H}^{1, g} Z(\tau)\right)\right) d \tau \\
& \leq M\left(t_{2}-t_{1}\right) \frac{t_{1}+t_{2}-t_{0}}{2}
\end{aligned}
$$

where $t_{0} \leq t_{1}<t_{2}$. Hence, $Z$ is Lipschitzian on $I_{Z}$ and consequently has a continuous extension $Z_{0}$ on $\left[t_{0}, c_{Z}\right]$. By continuity of $Z_{0}$, we get

$$
Z_{0}\left(c_{Z}\right)=I_{1}+\left(I_{2}\left(c_{Z}-t_{0}\right)+\int_{t_{0}}^{c_{Z}}\left(\int_{t_{0}}^{s} F\left(\tau, Z(\tau), D_{H}^{1, g} Z(\tau)\right) d \tau\right) d s\right) .
$$

This implies that $Z_{0}(t)$ is a (i-i)-solution of problem (3.1) on $\left[t_{0}, c_{Z}\right]$ and, clearly, $V\left(t, Z_{0}(t)\right.$, $\left.D_{H}^{1, g} Z_{0}(t)\right)<r(t), t \in\left[t_{0}, c_{Z}\right]$. Now, let $X_{1}=\lim _{t \rightarrow c^{-} Z} X\left(t, t_{0}, X_{0}, D_{H}^{1, g} X\left(t_{0}\right)\right), D_{H}^{1, g} X(t)_{1}=$ $\lim _{t \rightarrow c^{-} Z} D_{H}^{1, g} X\left(t, t_{0}, X_{0}\right)$ and consider the initial problem

$$
D_{H}^{2, g} X(t)=F\left(t, X(t), D_{H}^{1, g} X(t)\right), \quad X\left(c_{Z}\right)=X_{1}, \quad D_{H}^{1, g} X\left(c_{Z}\right)=X_{1}^{*} .
$$

The assumption of local existence implies that there exists a (i-i)-solution $X_{0}(t)$ on $\left[c_{Z}, c_{Z}+\right.$ $\delta), \delta>0$. Define

$$
Z_{1}(t)= \begin{cases}Z_{0}(t) & \text { for } t \in\left[t_{0}, c_{Z}\right] \\ X_{0}(t) & \text { for } t \in\left[c_{Z}, c_{Z}+\delta\right) .\end{cases}
$$


Therefore $Z_{1}(t)$ is a (i-i)-solution of problem (3.1) on $\left[t_{0}, c_{Z}+\delta\right)$, and, by repeating the arguments that were used to obtain (3.6), we get $V\left(t, Z_{1}(t), D_{H}^{1, g} Z_{1}(t)\right) \leq r(t), t \in\left[t_{0}, c_{Z}+\delta\right)$. This contradicts the maximality of $Z$, and hence $c_{Z}=+\infty$. The proof is complete.

Remark 3.1 In Theorem 3.3, if (3.5) is replaced by

$$
\begin{aligned}
\lim _{h \rightarrow 0^{+}} & \inf \frac{1}{h}\left[V\left(t+h, X \ominus(-1) h \int_{t_{0}}^{t} F\left(s, X, D_{H}^{1, g} X(t)\right) d s, D_{H}^{1, g} X(t)+h F\left(t, X, D_{H}^{1, g} X(t)\right)\right)\right. \\
& \left.-V\left(t, X, D_{H}^{1, g} X(t)\right)\right] \\
\leq & g\left(t, V(t, X), V^{\prime}(t, X)\right)
\end{aligned}
$$

then for every $X_{0}, D_{H}^{1, g} X\left(t_{0}\right) \in K_{C}(\mathbb{R})$ such that $V\left(t_{0}, X_{0}, D_{H}^{1, g} X\left(t_{0}\right)\right) \leq \max \left\{u_{0}, u_{0}^{\prime}\right\}$, problem (3.1) has a (ii-i)-solution $X(t)$ on $\left[t_{0}, \infty\right)$, which satisfies the estimate

$$
V\left(t, X, D_{H}^{1, g} X(t)\right) \leq r\left(t, t_{0}, u_{0}, u_{0}^{\prime}\right), \quad t \geq t_{0}
$$

Remark 3.2 In Theorem 3.3, if (3.5) is replaced by

$$
\begin{aligned}
\lim _{h \rightarrow 0^{+}} & \inf \frac{1}{h}\left[V\left(t+h, X+h \int_{t_{0}}^{t} F\left(s, X, D_{H}^{1, g} X(t)\right) d s, D_{H}^{1, g} X(t) \ominus(-1) h F\left(t, X, D_{H}^{1, g} X(t)\right)\right)\right. \\
& \left.-V\left(t, X, D_{H}^{1, g} X(t)\right)\right] \\
\leq & g\left(t, V(t, X), V^{\prime}(t, X)\right)
\end{aligned}
$$

then for every $X_{0}, D_{H}^{1, g} X\left(t_{0}\right) \in K_{C}(\mathbb{R})$ such that $V\left(t_{0}, X_{0}, D_{H}^{1, g} X\left(t_{0}\right)\right) \leq \max \left\{u_{0}, u_{0}^{\prime}\right\}$, problem (3.1) has a (i-ii)-solution $X(t)$ on $\left[t_{0}, \infty\right)$, which satisfies the estimate

$$
V\left(t, X, D_{H}^{1, g} X(t)\right) \leq r\left(t, t_{0}, u_{0}, u_{0}^{\prime}\right), \quad t \geq t_{0} .
$$

Remark 3.3 In Theorem 3.3, if (3.5) is replaced by

$$
\begin{aligned}
\lim _{h \rightarrow 0^{+}} \inf & \frac{1}{h}\left[V \left(t+h, X \ominus(-1) h \int_{t_{0}}^{t} F\left(s, X, D_{H}^{1, g} X(t)\right) d s,\right.\right. \\
& \left.\left.D_{H}^{1, g} X(t) \ominus(-1) h F\left(t, X, D_{H}^{1, g} X(t)\right)\right)-V\left(t, X, D_{H}^{1, g} X(t)\right)\right] \\
\leq & g\left(t, V(t, X), V^{\prime}(t, X)\right),
\end{aligned}
$$

then for every $X_{0}, D_{H}^{1, g} X\left(t_{0}\right) \in K_{C}(\mathbb{R})$ such that $V\left(t_{0}, X_{0}, D_{H}^{1, g} X\left(t_{0}\right)\right) \leq \max \left\{u_{0}, u_{0}^{\prime}\right\}$, problem (3.1) has a (ii-ii)-solution $X(t)$ on $\left[t_{0}, \infty\right)$, which satisfies the estimate

$$
V\left(t, X, D_{H}^{1, g} X(t)\right) \leq r\left(t, t_{0}, u_{0}, u_{0}^{\prime}\right), \quad t \geq t_{0}
$$

Proof One can obtain these results easily by using the same methods as in the proof of Theorem 3.3. 
In next theorem, we present the continuous dependence of the solution on the righthand side of the equation. First, let us again consider problem (3.1) of the form

$$
D_{H}^{2, g} X(t)=F_{1}\left(t, X(t), D_{H}^{1, g} X(t)\right), \quad X\left(t_{0}\right)=X_{0}, \quad D_{H}^{1, g} X\left(t_{0}\right)=X_{0}^{\prime},
$$

and a problem with another initial value and another right-hand side, i.e.,

$$
D_{H}^{2, g} X(t)=F_{2}\left(t, X(t), D_{H}^{1, g} X(t)\right), \quad X\left(t_{0}\right)=Y_{0}, \quad D_{H}^{1, g} X\left(t_{0}\right)=Y_{0}^{\prime} .
$$

Theorem 3.4 Assume that $X_{0}, Y_{0}, X_{0}^{\prime}, Y_{0}^{\prime} \in K_{C}(R)$ are nontrivial interval-valued variables and $F_{1}, F_{2}$ satisfy the conditions of Theorem 2.3. Let $X\left(t, X_{0}, X_{0}^{\prime}, F_{1}\right)$ and $X\left(t, Y_{0}, Y_{0}^{\prime}, F_{2}\right)$ be solutions of (3.10) and (3.11), respectively. Suppose further that there exist constants $\beta, \varepsilon$ such that

$$
H\left(F_{1}(t, A, B), F_{2}(t, A, B)\right) \leq \beta
$$

for every $(t, A, B) \in\left[t_{0}, T\right] \times K_{C}(\mathbb{R}) \times K_{C}(\mathbb{R})$. Then, for every solution $X\left(t, X_{0}, X_{0}^{\prime}, F_{1}\right)$ of $(3.10)$, the following estimation is true:

$$
\begin{aligned}
& H\left(X\left(t, X_{0}, X_{0}^{\prime}, F_{1}\right), X\left(t, Y_{0}, Y_{0}^{\prime}, F_{2}\right)\right) \\
& \quad \leq \frac{\left(\alpha_{1}+\alpha_{2} T+\frac{3}{2} \beta T^{2}\right)}{1-2 L\left(\frac{1-\exp \left(k\left(t_{0}-T\right)\right)-k\left(T-t_{0}\right) \exp \left(k\left(t_{0}-T\right)\right)}{k^{2}}+\frac{1-\exp \left(k\left(t_{0}-T\right)\right)}{k}\right)},
\end{aligned}
$$

where $\alpha_{1}=H\left(X_{0}, Y_{0}\right), \alpha_{2}=H\left(X_{0}^{\prime}, Y_{0}^{\prime}\right)$.

Proof Setting $X_{F_{1}}^{*}(t)=X\left(t, t_{0}, X_{0}, X_{0}^{\prime}, F_{1}\right)$ and $X_{F_{2}}^{*}(t)=X\left(t, t_{0}, Y_{0}, Y^{\prime}{ }_{0}, F_{2}\right)$, we obtain

$$
\begin{aligned}
H\left(X_{F_{1}}^{*}(t), X_{F_{2}}^{*}(t)\right) & \\
= & H\left(\begin{array}{l}
X_{0} \ominus(-1)\left(X_{0}^{\prime}\left(t-t_{0}\right) \ominus(-1) \int_{t_{0}}^{t}\left(\int_{t_{0}}^{s} F_{1}\left(\tau, X_{F_{1}}^{*}(\tau), D_{H}^{1, g}\left(X_{F_{1}}^{*}\right)(\tau)\right) d \tau\right) d s\right), \\
Y_{0} \ominus(-1)\left(Y^{\prime}{ }_{0}\left(t-t_{0}\right) \ominus(-1) \int_{t_{0}}^{t}\left(\int_{t_{0}}^{s} F_{2}\left(\tau, X_{F_{2}}^{*}(\tau), D_{H}^{1, g}\left(X_{F_{2}}^{*}\right)(\tau)\right) d \tau\right) d s\right)
\end{array}\right) \\
\leq & H\left(X_{0}, Y_{0}\right)+\left(t-t_{0}\right) H\left(X_{0}^{\prime}, Y_{0}^{\prime}\right) \\
& +H\left(\ominus(-1) \int_{t_{0}}^{t}\left(\int_{t_{0}}^{s} F_{1}\left(\tau, X_{F_{1}}^{*}(\tau), D_{H}^{1, g}\left(X_{F_{1}}^{*}\right)(\tau)\right) d \tau\right) d s,\right. \\
& \left.\ominus(-1) \int_{t_{0}}^{t}\left(\int_{t_{0}}^{s} F_{2}\left(\tau, X_{F_{2}}^{*}(\tau), D_{H}^{1, g}\left(X_{F_{2}}^{*}\right)(\tau)\right) d \tau\right) d s\right) \\
\leq & \alpha_{1}+\left(t-t_{0}\right) \alpha_{2} \\
& +\int_{t_{0}}^{t}\left(\int_{t_{0}}^{s} H\left(F_{1}\left(\tau, X_{F_{1}}^{*}(\tau), D_{H}^{1, g}\left(X_{F_{1}}^{*}\right)(\tau)\right), F_{2}\left(\tau, X_{F_{1}}^{*}(\tau), D_{H}^{1, g}\left(X_{F_{1}}^{*}\right)(\tau)\right)\right) d \tau\right) d s \\
& +\int_{t_{0}}^{t}\left(\int_{t_{0}}^{s} H\left(F_{2}\left(\tau, X_{F_{1}}^{*}(\tau), D_{H}^{1, g}\left(X_{F_{1}}^{*}\right)(\tau)\right), F_{2}\left(\tau, X_{F_{2}}^{*}(\tau), D_{H}^{1, g}\left(X_{F_{2}}^{*}\right)(\tau)\right)\right) d \tau\right) d s \\
\leq & \alpha_{1}+\left(t-t_{0}\right) \alpha_{2}+\frac{\beta t\left(t-t_{0}\right)}{2} \\
& +2 L \int_{t_{0}}^{t}\left(\int_{t_{0}}^{s}\left(H\left(X_{F_{1}}^{*}(\tau), X_{F_{2}}^{*}(\tau)\right)+H\left(D_{H}^{1, g}\left(X_{F_{1}}^{*}\right)(\tau), D_{H}^{1, g}\left(X_{F_{2}}^{*}\right)(\tau)\right)\right) d \tau\right) d s .
\end{aligned}
$$


On the other hand, we get

$$
\begin{aligned}
H\left(D_{H}^{1, g} X_{F_{1}}^{*}(t), D_{H}^{1, g} X_{F_{2}}^{*}(t)\right) \\
=H\left(\begin{array}{c}
X^{\prime}{ }_{0} \ominus(-1) \int_{t_{0}}^{t} F_{1}\left(s, X_{F_{1}}^{*}(s), D_{H}^{1, g} X_{F_{1}}^{*}(s)\right) d s, \\
Y^{\prime}{ }_{0} \ominus(-1) \int_{t_{0}}^{t} F_{1}\left(s, X_{F_{2}}^{*}(s), D_{H}^{1, g} X_{F_{2}}^{*}(s)\right) d s
\end{array}\right) \\
\leq \alpha_{2}+\beta\left(t-t_{0}\right) \\
\quad+2 L \int_{t_{0}}^{t}\left(H\left(X_{F_{1}}^{*}(s), X_{F_{2}}^{*}(s)\right)+H\left(D_{H}^{1, g} X_{F_{1}}^{*}(s), D_{H}^{1, g} X_{F_{2}}^{*}(s)\right)\right) d s .
\end{aligned}
$$

Combining (3.12) and (3.13), we have the estimate as follows:

$$
\begin{aligned}
& H_{0}^{1}\left(X_{F_{1}}^{*}(t), X_{F_{2}}^{*}(t)\right) \\
& \quad=H_{0}\left(X_{F_{1}}^{*}(t), X_{F_{2}}^{*}(t)\right)+H_{0}\left(D_{H}^{1, g} X_{F_{1}}^{*}(s), D_{H}^{1, g} X_{F_{2}}^{*}(s)\right) \\
& \quad \leq \frac{\left(\alpha_{1}+\alpha_{2} T+\frac{3}{2} \beta T^{2}\right)}{1-2 L\left(\frac{1-\exp \left(k\left(t_{0}-T\right)\right)-k\left(T-t_{0}\right) \exp \left(k\left(t_{0}-T\right)\right)}{k^{2}}+\frac{1-\exp \left(k\left(t_{0}-T\right)\right)}{k}\right)} .
\end{aligned}
$$

It is easily seen that a little change of initial conditions or a little change of the right-hand side of the equation causes a little change of corresponding solutions.

Next, we shall present some examples being simple illustrations of the theory of secondorder IDEs. Let us start the illustrations by considering the following second-order IDE:

$$
\left\{\begin{array}{l}
D_{H}^{2, g} X(t)+a D_{H}^{1, g} X(t)+b X(t)=K(t) \\
X\left(t_{0}\right)=I_{1} \\
D_{H}^{1, g} X\left(t_{0}\right)=I_{2}
\end{array}\right.
$$

where $a, b$ are positive constants, $I_{1}=\left[\underline{I}_{1}, \bar{I}_{1}\right], I_{2}=\left[\underline{I}_{2}, \bar{I}_{2}\right] \in K_{C}(\mathbb{R})$, and $K(t)=[\underline{K}(t), \bar{K}(t)]$ is a continuous interval-valued function on $\left[t_{0}, T\right]$, and $X(t)=[\underline{X}(t), \bar{X}(t)]$. Our strategy of solving (3.14) is based on the choice of the derivative in the interval-valued differential equation. In order to solve (3.14), we have three steps: first we choose the type of derivative and change problem (3.14) to a system of ODE by using Theorem 2.1 and considering initial values. Second we solve the obtained ODE system. The final step is to find such a domain in which the solution and its derivatives have valid sets, i.e., we ensure that $[\underline{X}(t), \bar{X}(t)],\left[(\underline{X})^{\prime}(t),(\bar{X})^{\prime}(t)\right]$ and $\left[(\underline{X})^{\prime \prime}(t),(\bar{X})^{\prime \prime}(t)\right]$ are valid sets.

By using Theorem 2.1, we obtain that four ODEs systems are possible for problem (3.14), as follows.

Case $1 X(t)$ and $D_{H}^{1, g} X(t)$ are (i)-differentiable

$$
\left\{\begin{array}{l}
\underline{X}^{\prime \prime}(t)+a \underline{X}^{\prime}(t)+b \underline{X}(t)=\underline{K}(t), \\
\bar{X}^{\prime \prime}(t)+a \bar{X}^{\prime}(t)+b \bar{X}(t)=\bar{K}(t), \\
\underline{X}\left(t_{0}\right)=\underline{I}_{1}, \quad \bar{X}\left(t_{0}\right)=\bar{I}_{1}, \\
\underline{X}^{\prime}\left(t_{0}\right)=\underline{I}_{2}, \quad \bar{X}^{\prime}\left(t_{0}\right)=\bar{I}_{2} .
\end{array}\right.
$$


Case $2 X(t)$ is (i)-differentiable and $D_{H}^{1, g} X(t)$ is (ii)-differentiable

$$
\left\{\begin{array}{l}
\bar{X}^{\prime \prime}(t)+a \underline{X}^{\prime}(t)+b \underline{X}(t)=\underline{K}(t), \\
\underline{X}^{\prime \prime}(t)+a \bar{X}^{\prime}(t)+b \bar{X}(t)=\overline{\bar{K}}(t), \\
\underline{X}\left(t_{0}\right)=\underline{I}_{1}, \quad \bar{X}\left(t_{0}\right)=\bar{I}_{1}, \\
\underline{X}^{\prime}\left(t_{0}\right)=\underline{I}_{2}, \quad \bar{X}^{\prime}\left(t_{0}\right)=\bar{I}_{2} .
\end{array}\right.
$$

Case $3 X(t)$ is (ii)-differentiable and $D_{H}^{1, g} X(t)$ is (i)-differentiable

$$
\left\{\begin{array}{l}
\bar{X}^{\prime \prime}(t)+a \bar{X}^{\prime}(t)+b \underline{X}(t)=\underline{K}(t), \\
\underline{X}^{\prime \prime}(t)+a \underline{X}^{\prime}(t)+b \bar{X}(t)=\overline{\bar{K}}(t), \\
\underline{X}\left(t_{0}\right)=\underline{I}_{1}, \quad \bar{X}\left(t_{0}\right)=\bar{I}_{1}, \\
\underline{X}^{\prime}\left(t_{0}\right)=\bar{I}_{2}, \quad \bar{X}^{\prime}\left(t_{0}\right)=\underline{I}_{2} .
\end{array}\right.
$$

Case $4 X(t)$ and $D_{H}^{1, g} X(t)$ are (ii)-differentiable

$$
\left\{\begin{array}{l}
\underline{X}^{\prime \prime}(t)+a \bar{X}^{\prime}(t)+b \underline{X}(t)=\underline{K}(t), \\
\bar{X}^{\prime \prime}(t)+a \underline{X^{\prime}}(t)+b \bar{X}(t)=\bar{K}(t), \\
\underline{X}\left(t_{0}\right)=\underline{I}_{1}, \quad \bar{X}\left(t_{0}\right)=\bar{I}_{1}, \\
\underline{X}^{\prime}\left(t_{0}\right)=\bar{I}_{2}, \quad \bar{X}^{\prime}\left(t_{0}\right)=\underline{I}_{2} .
\end{array}\right.
$$

Example 3.5 Let us consider the following interval-valued second-order differential equations:

$$
D_{H}^{2, g} X(t)=[-1,1], \quad X(0)=[-1,1], \quad D_{H}^{1, g} X(0)=[-1,1], \quad t \in[0,2] .
$$

Case 1: From (3.15), we get

$$
\left\{\begin{array}{l}
\underline{X}^{\prime \prime}(t)=-1, \\
\bar{X}^{\prime \prime}(t)=1, \\
\underline{X}(0)=-1, \quad \bar{X}(0)=1, \\
\underline{X}^{\prime}(0)=-1, \quad \bar{X}^{\prime}(0)=1 .
\end{array}\right.
$$

By solving (3.20), we obtain $X(t)=\left[-\left(t^{2} / 2+t+1\right),\left(t^{2} / 2+t+1\right)\right]$ and $X^{\prime}(t)$ are (i)differentiable. Hence, there is a solution in this case. This solution is shown in Figure 1.

Case 2: From (3.16), we have

$$
\begin{cases}\bar{X}^{\prime \prime}(t)=-1, & \\ \underline{X}^{\prime \prime}(t)=1, & \\ \underline{X}(0)=-1, & \bar{X}(0)=1, \\ \underline{X}^{\prime}(0)=-1, & \bar{X}^{\prime}(0)=1 .\end{cases}
$$

By solving (3.21), we get $X(t)=\left[-\left(-t^{2} / 2+t+1\right),\left(-t^{2} / 2+t+1\right)\right]$ is not (i)-differentiable, there is no solution in this case. 
Figure 1 Solution of Example 3.5 in Case 1.

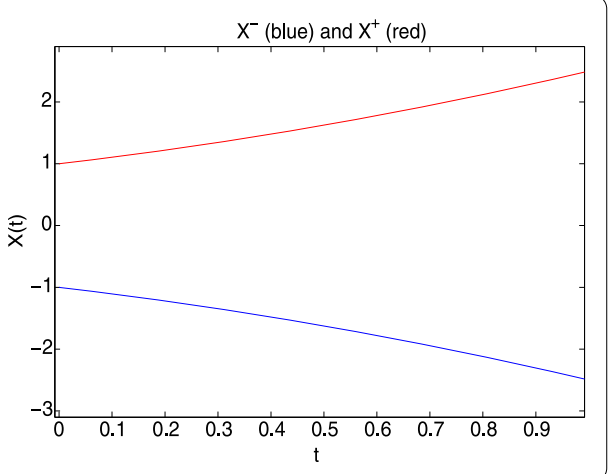

Figure 2 Solution of Example 3.5 in Case 3.

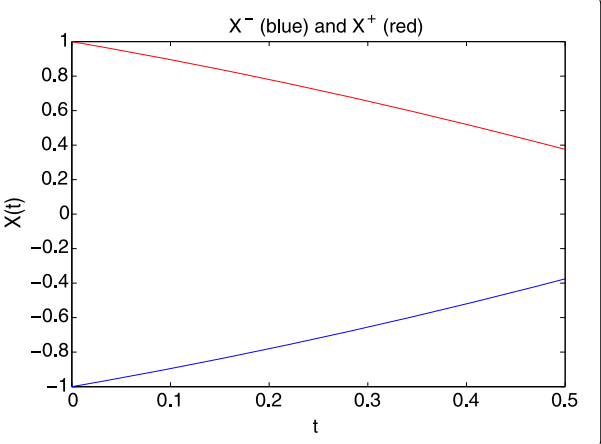

Case 3: From (3.17), we obtain

$$
\left\{\begin{array}{l}
\underline{X}^{\prime \prime}(t)=-1, \\
\bar{X}^{\prime \prime}(t)=1, \\
\underline{X}(0)=-1, \quad \bar{X}(0)=1, \\
\underline{X}^{\prime}(0)=1, \quad \bar{X}^{\prime}(0)=-1 .
\end{array}\right.
$$

By solving (3.22), we get $X(t)=\left[-\left(-t^{2} / 2-t+1\right),\left(-t^{2} / 2-t+1\right)\right]$. Notice that, in this case, since $X(t)$ is (ii)-differentiable and $X^{\prime}(t)$ is (i)-differentiable, such a solution is acceptable. This solution is shown in Figure 2.

Case 4: From (3.18), we have

$$
\left\{\begin{array}{l}
\bar{X}^{\prime \prime}(t)=-1, \\
\underline{X}^{\prime \prime}(t)=1, \\
\underline{X}(0)=-1, \quad \bar{X}(0)=1, \\
\underline{X}^{\prime}(0)=1, \quad \bar{X}^{\prime}(0)=-1 .
\end{array}\right.
$$

By solving (3.23), we have $X(t)=\left[-\left(t^{2} / 2-t+1\right),\left(t^{2} / 2-t+1\right)\right]$ is not (ii)-differentiable on $(1,2]$. Therefore, there is no solution in this case.

Example 3.6 Let us consider the following interval-valued second-order differential equations:

$$
\begin{aligned}
& D_{H}^{2, g} X(t)+X(t)=[0,2], \quad X(0)=[-1,1], \\
& D_{H}^{1, g} X(0)=[-1,1], \quad t \in[0, \pi / 4] .
\end{aligned}
$$


Case 1: From (3.15), we get

$$
\left\{\begin{array}{l}
\underline{X}^{\prime \prime}(t)+\underline{X}(t)=0, \\
\bar{X}^{\prime \prime}(t)+\bar{X}(t)=2, \\
\underline{X}(0)=-1, \quad \bar{X}(0)=1, \\
\underline{X}^{\prime}(0)=-1, \quad \bar{X}^{\prime}(0)=1 .
\end{array}\right.
$$

By solving (3.25), we obtain $X(t)=[-(\sin (t)+\cos (t)), 2+\sin (t)-\cos (t)]$. Since $X^{\prime}(t)$ is not (i)-differentiable, there is no solution in this case.

Case 2: From (3.16), we have

$$
\left\{\begin{array}{l}
\bar{X}^{\prime \prime}(t)+\underline{X}(t)=0, \\
\underline{X}^{\prime \prime}(t)+\overline{\bar{X}}(t)=2, \\
\underline{X}(0)=-1, \quad \bar{X}(0)=1, \\
\underline{X}^{\prime}(0)=-1, \quad \bar{X}^{\prime}(0)=1 .
\end{array}\right.
$$

By solving (3.26), we get $X(t)=[-(\exp (t)-\exp (-t)) / 2-\cos (t), 2+(\exp (t)-\exp (-t)) / 2-$ $\cos (t)]$. Since, $X^{\prime}(t)$ is not (ii)-differentiable, there is no solution in this case.

Case 3: From (3.17), we obtain

$$
\left\{\begin{array}{l}
\underline{X}^{\prime \prime}(t)+\underline{X}(t)=0, \\
\bar{X}^{\prime \prime}(t)+\bar{X}(t)=2, \\
\underline{X}(0)=-1, \quad \bar{X}(0)=1, \\
\underline{X}^{\prime}(0)=1, \quad \bar{X}^{\prime}(0)=-1 .
\end{array}\right.
$$

By solving (3.27), we get $X(t)=[(\exp (t)-\exp (-t)) / 2-\cos (t), 2-(\exp (t)-\exp (-t)) / 2-$ $\cos (t)]$. Notice that, in this case, since $X(t)$ is (ii)-differentiable and $X^{\prime}(t)$ is (i)-differentiable, such a solution is acceptable. This solution is shown in Figure 3.

Case 4: From (3.18), we have

$$
\left\{\begin{array}{l}
\bar{X}^{\prime \prime}(t)+\underline{X}(t)=0, \\
\underline{X}^{\prime \prime}(t)+\overline{\bar{X}}(t)=2, \\
\underline{X}(0)=-1, \quad \bar{X}(0)=1, \\
\underline{X}^{\prime}(0)=1, \quad \bar{X}^{\prime}(0)=-1 .
\end{array}\right.
$$

By solving (3.28), we have $X(t)=[\sin (t)-\cos (t), 2-\sin (t)-\cos (t)]$ and $X^{\prime}(t)$ are (ii)differentiable. Therefore, the obtained solution is valid. This solution is shown in Figure 4 .

Figure 3 Solution of Example 3.6 in Case 3.

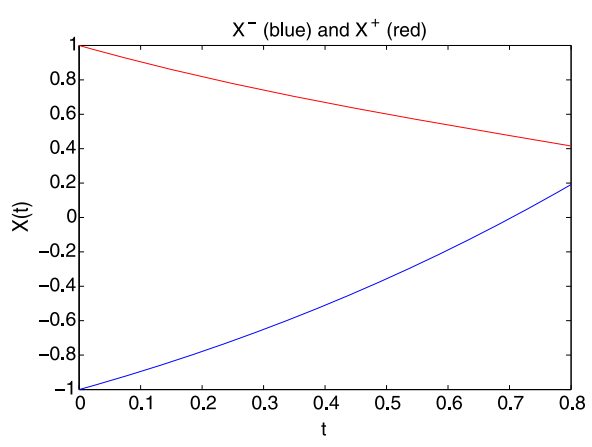


Figure 4 Solution of Example 3.6 in Case 4.

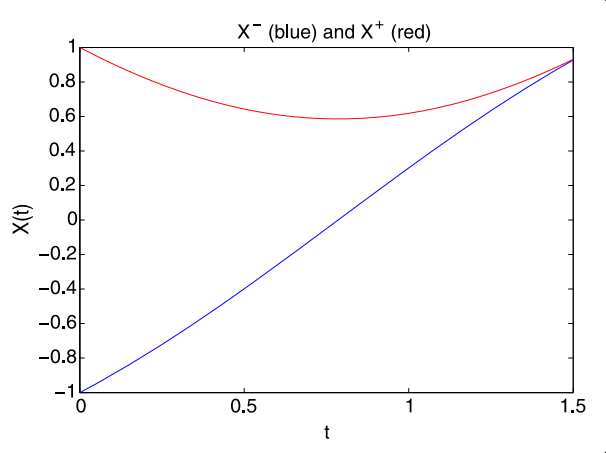

\section{A new method for solving interval-valued second-order differential equations}

In [48] the author applied the modification of Laplace decomposition method (LDM) to solve nonlinear interval-valued Volterra integral equations. The modified LDM can be applied to derive the lower and upper solutions. In [46] Salahshour and Allahviranloo solved the second-order fuzzy differential equation under generalized H-differentiability by using fuzzy Laplace transforms. Now, in Section 3 we see that some second-order IDEs are solved under generalized H-differentiability. For the second-order IDEs, there exist at most four solutions, but all the obtained solutions may not be acceptable. It is an important result in the theory of IDEs of higher order. Notice that by applying the above approach (in Section 3), the original second-order IDEs are transformed to four ordinary differential systems. However, by applying Salahshour et al.'s [46] approach, each solution can be obtained directly. In this section, we propose a new algorithm based on the analysis of a crisp solution. We establish a synthesis of a crisp solution of an interval-valued initial value problem and the method proposed in Kaleva [5] and Akin [50].

Let us again consider the interval-valued second-order differential equations of the form

$$
A D_{H}^{2, g} X(t)+B D_{H}^{1, g} X(t)+C X(t)=F(t), \quad X\left(t_{0}\right)=I_{1}, \quad D_{H}^{1, g} X\left(t_{0}\right)=I_{2},
$$

where $A, B, C$ are interval-valued constants in $K_{C}(\mathbb{R})$ and $X(t), F(t)$ are interval-valued functions. We define $A=[\underline{A}, \bar{A}], B=[\underline{B}, \bar{B}], C=[\underline{C}, \bar{C}]$ and $X(t)=[\underline{X}(t), \bar{X}(t)], F(t)=$ $[\underline{F}(t), \bar{F}(t)]$. Hence we obtain from $(4.1)$

$$
[\underline{A}, \bar{A}]\left[\underline{X}^{\prime \prime}(t), \bar{X}^{\prime \prime}(t)\right]+[\underline{B}, \bar{B}]\left[\underline{X}^{\prime}(t), \bar{X}^{\prime}(t)\right]+[\underline{C}, \bar{C}][\underline{X}(t), \bar{X}(t)]=[\underline{F}(t), \bar{F}(t)] .
$$

Then we have

$$
\min \left\{[\underline{A}, \bar{A}]\left[\underline{X}^{\prime \prime}(t), \bar{X}^{\prime \prime}(t)\right]+[\underline{B}, \bar{B}]\left[\underline{X}^{\prime}(t), \bar{X}^{\prime}(t)\right]+[\underline{C}, \bar{C}][\underline{X}(t), \bar{X}(t)]\right\}=\underline{F}(t)
$$

and

$$
\max \left\{[\underline{A}, \bar{A}]\left[\underline{X}^{\prime \prime}(t), \bar{X}^{\prime \prime}(t)\right]+[\underline{B}, \bar{B}]\left[\underline{X}^{\prime}(t), \bar{X}^{\prime}(t)\right]+[\underline{C}, \bar{C}][\underline{X}(t), \bar{X}(t)]\right\}=\bar{F}(t) .
$$

From two min and max problems, we notice that it is not an easy task to determine the result of the min and max problems, so we propose the following method. 
Step 1: Problem (4.1) is considered as a crisp problem and solved. Here we chose the crisp problem

$$
\begin{aligned}
& a x^{\prime \prime}(t)+b x^{\prime}(t)+c x(t)=f(t), \\
& x\left(t_{0}\right)=I_{1}^{*}, \quad x^{\prime}\left(t_{0}\right)=I_{2}^{*},
\end{aligned}
$$

where $x(t)$ is a real-valued solution of (4.3), $a \in A, b \in B, c \in C, I_{1}^{*} \in I_{1}$ and $I_{2}^{*} \in I_{2}$.

Step 2: Next, we investigate the behavior of the crisp solution $x(t)$ in (4.3) and the following four cases according to domains:

+ Let the domain where $x^{\prime}(t) \geq 0, x^{\prime \prime}(t) \geq 0$ be $\mathcal{D}_{1}$.

+ Let the domain where $x^{\prime}(t) \geq 0, x^{\prime \prime}(t) \leq 0$ be $\mathcal{D}_{2}$.

+ Let the domain where $x^{\prime}(t) \leq 0, x^{\prime \prime}(t) \leq 0$ be $\mathcal{D}_{3}$.

+ Let the domain where $x^{\prime}(t) \leq 0, x^{\prime \prime}(t) \geq 0$ be $\mathcal{D}_{4}$.

Step 3: After we obtain the four domains above, we set:

$+[\underline{X}(t), \bar{X}(t)],\left[\underline{X}^{\prime}(t), \bar{X}^{\prime}(t)\right]$ and $\left[\underline{X}^{\prime \prime}(t), \bar{X}^{\prime \prime}(t)\right]$ in domain $\mathcal{D}_{1}$.

$+[\underline{X}(t), \bar{X}(t)],\left[\underline{X}^{\prime}(t), \bar{X}^{\prime}(t)\right]$ and $\left[\bar{X}^{\prime \prime}(t), \underline{X}^{\prime \prime}(t)\right]$ in domain $\mathcal{D}_{2}$.

$+[\underline{X}(t), \bar{X}(t)],\left[\bar{X}^{\prime}(t), \underline{X}^{\prime}(t)\right]$ and $\left[\bar{X}^{\prime \prime}(t), \underline{X}^{\prime \prime}(t)\right]$ in domain $\mathcal{D}_{3}$.

$+[\underline{X}(t), \bar{X}(t)],\left[\bar{X}^{\prime}(t), \underline{X}^{\prime}(t)\right]$ and $\left[\underline{X}^{\prime \prime}(t), \bar{X}^{\prime \prime}(t)\right]$ in domain $\mathcal{D}_{4}$.

Step 4: Substitute the obtained domains in (4.1). Then we obtain that four ODEs systems are possible for problem (4.1), as follows:

Case 1: In domain $\mathcal{D}_{1}$

$$
\left\{\begin{array}{l}
{[\underline{A}, \bar{A}]\left[\underline{X}^{\prime \prime}(t), \bar{X}^{\prime \prime}(t)\right]+[\underline{B}, \bar{B}]\left[\underline{X}^{\prime}(t), \bar{X}^{\prime}(t)\right]} \\
\quad+[\underline{C}, \bar{C}][\underline{X}(t), \bar{X}(t)]=[\underline{F}(t), \bar{F}(t)], \\
{\left[\underline{X}\left(t_{0}\right), \bar{X}\left(t_{0}\right)\right]=\left[\underline{I}_{1}, \bar{I}_{1}\right]} \\
{\left[\underline{X}^{\prime}\left(t_{0}\right), \bar{X}^{\prime}\left(t_{0}\right)\right]=\left[\underline{I}_{2}, \bar{I}_{2}\right] .}
\end{array}\right.
$$

Case 2: In domain $\mathcal{D}_{2}$

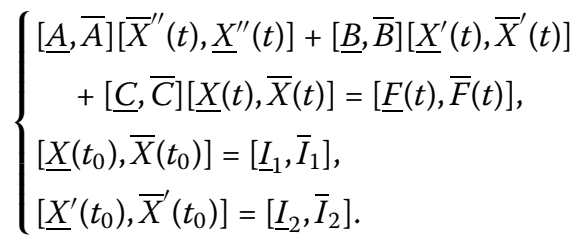

Case 3: In domain $\mathcal{D}_{3}$

$$
\left\{\begin{array}{l}
{[\underline{A}, \bar{A}]\left[\bar{X}^{\prime \prime}(t), \underline{X}^{\prime \prime}(t)\right]+[\underline{B}, \bar{B}]\left[\bar{X}^{\prime}(t), \underline{X}^{\prime}(t)\right]} \\
\quad+[\underline{C}, \bar{C}][\underline{X}(t), \bar{X}(t)]=[\underline{F}(t), \bar{F}(t)], \\
{\left[\underline{X}\left(t_{0}\right), \bar{X}\left(t_{0}\right)\right]=\left[\underline{I}_{1}, \bar{I}_{1}\right]} \\
{\left[\bar{X}^{\prime}\left(t_{0}\right), \underline{X}^{\prime}\left(t_{0}\right)\right]=\left[\underline{I}_{2}, \bar{I}_{2}\right] .}
\end{array}\right.
$$


Case 4: In domain $\mathcal{D}_{4}$

$$
\left\{\begin{array}{l}
{[\underline{A}, \bar{A}]\left[\underline{X}^{\prime \prime}(t), \bar{X}^{\prime \prime}(t)\right]+[\underline{B}, \bar{B}]\left[\bar{X}^{\prime}(t), \underline{X}^{\prime}(t)\right]} \\
\quad+[\underline{C}, \bar{C}][\underline{X}(t), \bar{X}(t)]=[\underline{F}(t), \bar{F}(t)], \\
{\left[\underline{X}\left(t_{0}\right), \bar{X}\left(t_{0}\right)\right]=\left[\underline{I}_{1}, \bar{I}_{1}\right]} \\
{\left[\bar{X}^{\prime}\left(t_{0}\right), \underline{X}^{\prime}\left(t_{0}\right)\right]=\left[\underline{I}_{2}, \bar{I}_{2}\right] .}
\end{array}\right.
$$

Example 4.1 Consider the following interval-valued second-order differential equation with initial condition:

$$
[2,4] D_{H}^{2, g} X(t)=[0,2], \quad X(0)=[1,3], \quad D_{H}^{1, g} X(0)=[1,3] .
$$

Now, before solving ISDE (4.8), let us solve a crisp problem corresponding to (4.8), to investigate the behavior of a solution. The crisp problem is

$$
3 x^{\prime \prime}(t)=1, \quad x(0)=2, \quad x^{\prime}(0)=2 .
$$

A solution for (4.9) is $x(t)=(1 / 6) t^{2}+2 t+2$. The derivatives of a crisp solution are $x^{\prime}(t)=$ $(1 / 3) t+2$ and $x^{\prime \prime}(t)=1 / 3$. So, for $t \geq-6, x^{\prime}(t) \geq 0$ and for any $t, x^{\prime \prime}(t) \geq 0$. Therefore $\mathcal{D}_{1}=$ $\{t \in \mathbb{R} \mid t \geq-6\}$. Similarly, we have $D_{4}=\{t \in \mathbb{R} \mid t \leq-6\}$. Now, if we substitute the obtained domains in (4.8) for $t \in \mathcal{D}_{1}$, we get the following:

$$
\left\{\begin{array}{l}
{[2,4]\left[\underline{X}^{\prime \prime}(t), \bar{X}^{\prime \prime}(t)\right]=[0,2],} \\
{[\underline{X}(0), \bar{X}(0)]=[1,3]} \\
{\left[\underline{X}^{\prime}(0), \bar{X}^{\prime}(0)\right]=[1,3] .}
\end{array}\right.
$$

Then the solution for (4.10) is as follows:

$$
\left\{\begin{array}{l}
\underline{X}(t)=t+1, \\
\bar{X}(t)=\frac{t^{2}}{4}+3 t+3 .
\end{array}\right.
$$

Next we must ensure that $\bar{X}(t) \geq \underline{X}(t), \bar{X}^{\prime}(t) \geq \underline{X}^{\prime}(t)$ and $\bar{X}^{\prime \prime}(t) \geq \underline{X^{\prime \prime}}(t)$ for all $t \geq-6$. But $\bar{X}(t) \leq \underline{X}(t)$ for any $t \in[-6,-4+2 \sqrt{2}]$ and $\bar{X}^{\prime}(t) \leq \underline{X}^{\prime}(t)$ for any $t \leq-4$. So (4.11) cannot be a solution for (4.8) according to the proposed method. We can see Figure 5. Similarly, we can do the same process for $t \in \mathcal{D}_{4}$. If we substitute the obtained in (4.8) for $t \in \mathcal{D}_{4}$, we get the following:

$$
\left\{\begin{array}{l}
{[2,4]\left[\underline{X}^{\prime \prime}(t), \bar{X}^{\prime \prime}(t)\right]=[0,2]} \\
{\left[\underline{X}^{\prime}(0), \bar{X}(0)\right]=[1,3]} \\
{\left[\bar{X}^{\prime}(0), \underline{X}^{\prime}(0)\right]=[1,3]}
\end{array}\right.
$$

Then the solution for (4.12) is as follows:

$$
\left\{\begin{array}{l}
\underline{X}(t)=3 t+1, \\
\bar{X}(t)=\frac{t^{2}}{4}+t+3 .
\end{array}\right.
$$


Figure 5 Solution of Example 4.1.

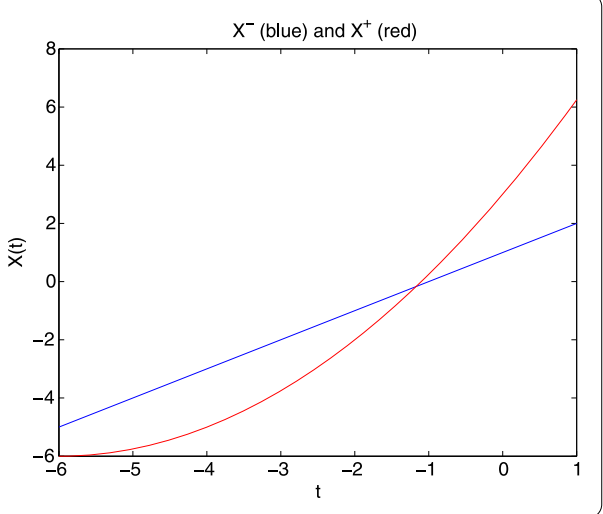

Figure 6 Solution of Example 4.1.

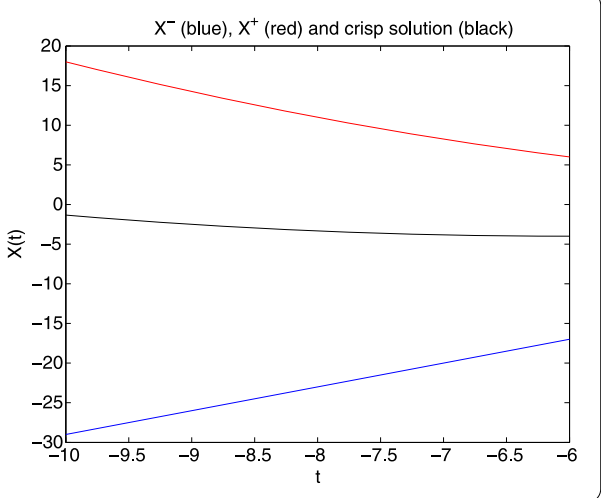

After necessary computations, we see that $\bar{X}(t) \geq \underline{X}(t), \bar{X}^{\prime}(t) \geq \underline{X^{\prime}}(t)$ and $\bar{X}^{\prime \prime}(t) \geq \underline{X}^{\prime \prime}(t)$ for all $t \leq-6$. So, (4.13) is a solution for (4.8) according to the proposed method. In Figure 6, solution curves of (4.8) are given.

Example 4.2 Consider the following interval-valued second-order differential equation with initial condition:

$$
D_{H}^{2, g} X(t)=2 D_{H}^{1, g} X(t), \quad X(0)=[2,4], \quad D_{H}^{1, g} X(0)=[1,3] .
$$

Now, before solving ISDE (4.14), let us solve a crisp problem corresponding to (4.14), to investigate the behavior of a solution. The crisp problem is

$$
x^{\prime \prime}(t)=2 x^{\prime}(t), \quad x(0)=3, \quad x^{\prime}(0)=2 .
$$

A solution for (4.15) is $x(t)=2+\exp (2 t)$. The derivatives of the crisp solution are $x^{\prime}(t)=$ $2 \exp (2 t)$ and $x^{\prime \prime}(t)=4 \exp (2 t)$. So, for any $t, x^{\prime}(t)>0$ and for any $t, x^{\prime \prime}(t)>0$. Therefore $\mathcal{D}_{1}=\mathbb{R}$. Now, if we substitute the obtained domain in (4.14) for $t \in \mathcal{D}_{1}$, we get the following:

$$
\left\{\begin{array}{l}
{\left[\underline{X}^{\prime \prime}(t), \bar{X}^{\prime \prime}(t)\right]=2\left[\underline{X}^{\prime}(t), \bar{X}^{\prime}(t)\right],} \\
{[\underline{X}(0), \bar{X}(0)]=[2,4],} \\
{\left[\underline{X}^{\prime}(0), \bar{X}^{\prime}(0)\right]=[1,3] .}
\end{array}\right.
$$


Figure 7 Solution of Example 4.2.

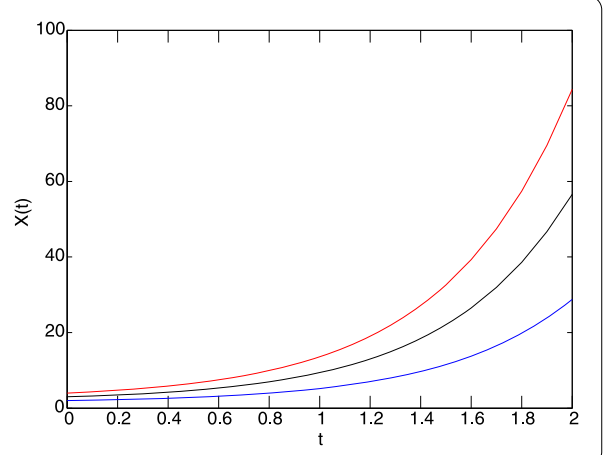

Then the solution for (4.16) is as follows:

$$
\left\{\begin{array}{l}
\underline{X}(t)=\frac{3}{2}+\frac{1}{2} \exp (2 t), \\
\bar{X}(t)=\frac{5}{2}+\frac{3}{2} \exp (2 t) .
\end{array}\right.
$$

Similarly as in Example 4.1, we must ensure that $\bar{X}(t) \geq \underline{X}(t), \bar{X}^{\prime}(t) \geq \underline{X}^{\prime}(t)$ and $\bar{X}^{\prime \prime}(t) \geq$ $\underline{X}^{\prime \prime}(t)$. We obtain $\bar{X}^{\prime}(t)=3 \exp (2 t), \underline{X}^{\prime}(t)=\exp (2 t)$ and $\bar{X}^{\prime \prime}(t)=6 \exp (2 t), \underline{X}^{\prime \prime}(t)=2 \exp (2 t)$. Therefore (4.17) is a solution for (4.14) for any $t$. We can see Figure $7(\underline{X}(t)$ (blue), $\bar{X}(t)$ (red) and the crisp solution (black)).

\section{Conclusions}

In this paper, we have obtained a global existence result for a solution to interval-valued second-order differential equations. Also, we have proposed a new algorithm based on the analysis of a crisp solution. The proposed method in this paper may be useful if the coefficients, initial values and forcing terms are interval. If the crisp problem is not solvable explicitly, we cannot determine the mentioned domains precisely.

Competing interests

The authors declare that they have no competing interests.

Authors' contributions

All authors read and approved the final version of the manuscript.

\section{Author details}

'Division of Applied Mathematics, Ton Duc Thang University, Nguyen Huu Tho Street, District 7, Ho Chi Minh City,

Vietnam. ${ }^{2}$ Faculty of Mathematics and Computer Science, University of Science, VNU, Ho Chi Minh City, Vietnam.

\section{Acknowledgements}

The authors would like to express their gratitude to the anonymous referees for their helpful comments and suggestions, which have greatly improved the paper.

Received: 18 April 2013 Accepted: 27 August 2013 Published: 07 Nov 2013

\section{References}

1. De Blasi, FS, lervolino, F: Equazioni differenziali con soluzioni a valore compatto convesso. Boll. Unione Mat. Ital. 4(2), 491-501 (1969)

2. Agarwal, RP, O'Regan, D, Lakshmikantham, V: Viability theory and fuzzy differential equations. Fuzzy Sets Syst. 151(3), 563-580 (2005)

3. Agarwal, RP, O'Regan, D, Lakshmikantham, V: A stacking theorem approach for fuzzy differential equations. Nonlinear Anal. TMA 55(3), 299-312 (2003)

4. Ngo, VH, Nguyen, DP: On maximal and minimal solutions for set-valued differential equations with feedback control. Abstr. Appl. Anal. (2012). doi:10.1155/2012/816218

5. Kaleva, O: Fuzzy differential equations. Fuzzy Sets Syst. 24, 301-317 (1987)

6. Lakshmikantham, V, Bhaskar, TG, Devi, JV: Theory of Set Differential Equations in Metric Spaces. Cambridge Scientific Publisher, Cambridge (2006) 
7. De Blasi, FS, Lakshmikantham, V, Bhaskar, TG: An existence theorem for set differential inclusions in a semilinear metric space. Control Cybern. 36(3), 571-582 (2007)

8. Song, S, Wu, C: Existence and uniqueness of solutions to Cauchy problem of fuzzy differential equations. Fuzzy Sets Syst. 110, 55-67 (2000)

9. Bhaskar, TG, Lakshmikantham, V: Set differential equations and flow invariance. J. Appl. Anal. 82(2), 357-368 (2003)

10. Agarwal, RP, O'Regan, D: Existence for set differential equations via multivalued operator equations. In: Differential Equations and Applications, vol. 5, pp. 1-5 (2007)

11. Truong, VA, Ngo, VH, Nguyen, DP: A note on solutions of interval-valued Volterra integral equations. J. Integral Equ. Appl. (2013, in press)

12. Stefanini, L, Bede, B: Generalized Hukuhara differentiability of interval-valued functions and interval differential equations. Nonlinear Anal. TMA 71, 1311-1328 (2009). doi:10.1016/j.na.2008.12.005

13. Chalco-Cano, Y, Rufián-Lizana, A, Román-Flores, H, Jiménez-Gamero, MD: Calculus for interval-valued functions using generalized Hukuhara derivative and applications. Fuzzy Sets Syst. (2012). doi:10.1016/j.fss.2012.12.004

14. Malinowski, MT: Interval Cauchy problem with a second type Hukuhara derivative. Inf. Sci. 213, $94-105$ (2012). doi:10.1016/j.ins.2012.05.022

15. Malinowski, MT: Interval differential equations with a second type Hukuhara derivative. Appl. Math. Lett. 24 2118-2123 (2011)

16. Truong, VA, Ngo, VH, Nguyen, DP: Global existence of solutions for interval-valued integro-differential equations under generalized H-differentiability. Adv. Differ. Equ. 2013, 217 (2013). doi:10.1186/1687-1847-2013-217

17. Moore, R, Lodwick, W: Interval analysis and fuzzy set theory. Fuzzy Sets Syst. 135(1), 5-9 (2003)

18. Georgiou, DN, Nieto, JJ, Rodríguez-López, R: Initial value problems for higher-order fuzzy differential equations. Nonlinear Anal. 63, 587-600 (2005)

19. Bede, B, Stefanini, L: Generalized differentiability of fuzzy-valued functions. Fuzzy Sets Syst. (2012). doi:10.1016/j.fss.2012.10.003

20. Allahviranloo, T, Khezerloo, M, Sedaghatfar, O, Salahshour, S: Toward the existence and uniqueness of solutions of second-order fuzzy Volterra integro-differential equations with fuzzy kernel. Neural Comput. Appl. 22, S133-S141 (2013)

21. Salahshour, S: Nth-order fuzzy differential equations under generalized differentiability. J. Fuzzy Set Valued Anal. 2011, Article ID jfsva-00043 (2011)

22. Rabiei, F, Ismail, F, Ahmadian, A, Salahshour, S: Numerical solution of second-order fuzzy differential equation using improved Runge-Kutta Nystrom method. Math. Probl. Eng. 2013, Article ID 803462 (2013)

23. Allahviranloo, T, Amirteimoori, A, Khezerloo, M, Khezerloo, S: A new method for solving fuzzy Volterra integro-differential equations. Aust. J. Basic Appl. Sci. 5(4), 154-164 (2011).

24. Alikhani, R, Bahrami, F, Jabbari, A: Existence of global solutions to nonlinear fuzzy Volterra integro-differential equations. Nonlinear Anal. TMA 75(4), 1810-1821 (2012)

25. Bede, B, Gal, SG: Generalizations of the differentiability of fuzzy-number-valued functions with applications to fuzzy differential equations. Fuzzy Sets Syst. 151, 581-599 (2005)

26. Bede, B, Rudas, IG, Bencsik, AL: First order linear fuzzy differential equations under generalized differentiability. Inf. Sci. $177,1648-1662$ (2007)

27. Chalco-Cano, Y, Román-Flores, H: On new solutions of fuzzy differential equations. Chaos Solitons Fractals 38 $112-119(2008)$

28. Stefanini, L, Sorini, L, Guerra, ML: Parametric representation of fuzzy numbers and application to fuzzy calculus. Fuzzy Sets Syst. 157, 2423-2455 (2006)

29. Stefanini, L: A generalization of Hukuhara difference. In: Dubois, D, Lubiano, MA, Prade, H, Gil, MA, Grzegorzewski, P, Hryniewicz, O (eds.) Soft Methods for Handling Variability and Imprecision. Series on Advances in Soft Computing, vol. 48. Springer, Berlin (2008)

30. Nguyen, DP, Ngo, VH, Ho, V: On comparisons of set solutions for fuzzy control integro-differential systems. J. Adv. Res. Appl. Math. 4(1), 84-101 (2012). doi:10.5373/jaram

31. Mizukoshi, MT, Barros, LC, Chalco-Cano, Y, Román-Flores, H, Bassanezi, RC: Fuzzy differential equations and the extension principle. Inf. Sci. 177, 3627-3635 (2007)

32. Malinowski, MT: Second type Hukuhara differentiable solutions to the delay set-valued differential equations. Appl. Math. Comput. 218(18), 9427-9437 (2012)

33. Zhang, D, Feng, W, Zhao, Y, Qiu, J: Global existence of solutions for fuzzy second-order differential equations under generalized H-differentiability. Comput. Math. Appl. 60, 1548-1556 (2010)

34. Ahmadian, A, Suleiman, M, Salahshour, S, Baleanu, D: A Jacobi operational matrix for solving a fuzzy linear fractional differential equation. Adv. Differ. Equ. 2013, 104 (2013)

35. Allahviranloo, T, Salahshour, S, Homayoun-nejad, M, Baleanu, D: General solutions of fully fuzzy linear systems. Abstr. Appl. Anal. 2013, Article ID 593274 (2013)

36. Ngo, VH, Nguyen, DP: Fuzzy functional integro-differential equations under generalized H-differentiability. J. Intell. Fuzzy Syst. (in press). doi:10.3233/IFS-130883

37. Ho, V, Ngo, VH, Nguyen, DP: The local existence of solutions for random fuzzy integro-differential equations under generalized H-differentiability. J. Intell. Fuzzy Syst. (in press). doi:10.3233/IFS-130940

38. Malinowski, MT: Random fuzzy differential equations under generalized Lipschitz condition. Nonlinear Anal., Real World Appl. 13(2), 860-881 (2012)

39. Malinowski, MT: Existence theorems for solutions to random fuzzy differential equations. Nonlinear Anal., Theory Methods Appl. 73(6), 1515-1532 (2010)

40. Malinowski, MT: On random fuzzy differential equations. Fuzzy Sets Syst. 160(21), 3152-3165 (2009)

41. Allahviranloo, T, Hajighasemi, S, Khezerloo, M, Khorasany, M, Salahshour, S: Existence and uniqueness of solutions of fuzzy Volterra integro-differential equations. In: IPMU 2010, Part II. CCIS, vol. 81, pp. 491-500 (2010)

42. Allahviranloo, T, Salahshour, S, Abbasbandy, S: Solving fuzzy fractional differential equations by fuzzy Laplace transforms. Commun. Nonlinear Sci. Numer. Simul. 17(3), 1372-1381 (2012). doi:10.1016/j.cnsns.2011.07.005 
43. Allahviranloo, T, Abbasbandy, S, Sedaghatfar, O, Darabi, $P$ : A new method for solving fuzzy integro-differential equation under generalized differentiability. Neural Comput. Appl. 21(1), 191-196 (2012) doi:10.1007/s00521-011-0759-3

44. Allahviranloo, T, Salahshour, S: A new approach for solving first order fuzzy differential equation. In: Information Processing and Management of Uncertainty in Knowledge-Based Systems. Applications Communications in Computer and Information Science, vol. 81, pp. 522-531 (2010)

45. Allahviranloo, T, Salahshour, S: Euler method for solving hybrid fuzzy differential equation. Soft Comput. 15, 1247-1253 (2011)

46. Salahshour, S, Allahviranloo, T: Applications of fuzzy Laplace transforms. Soft Comput. 17, 145-158 (2013)

47. Salahshour, S, Allahviranloo, T: Application of fuzzy differential transform method for solving fuzzy Volterra integral equations. Appl. Math. Model. 37, 1016-1027 (2013)

48. Salahshour, S, Khan, M: Exact solutions of nonlinear interval Volterra integral equations. Int. J. Ind. Math. 4, Article ID IJIM-00291 (2012)

49. Salahshour, S, Khezerloo, M, Hajighasemi, S, Khorasany, M: Solving fuzzy integral equations of the second kind by fuzzy Laplace transform method. Int. J. Ind. Math. 4, 21-29 (2012)

50. Akin, Ö, Khaniyev, T, Oruc, Ö, Türksen, IB: An algorithm for the solution of second order fuzzy initial value problems. Expert Syst. Appl. 40, 953-957 (2013)

10.1186/1687-1847-2013-290

Cite this article as: Ngo and Nguyen: Global existence of solutions for interval-valued second-order differential equations under generalized Hukuhara derivative. Advances in Difference Equations 2013, 2013:290

\section{Submit your manuscript to a SpringerOpen ${ }^{\circ}$ journal and benefit from:}

- Convenient online submission

- Rigorous peer review

- Immediate publication on acceptance

- Open access: articles freely available online

- High visibility within the field

- Retaining the copyright to your article 